\title{
Principle of Limiting Factors-Driven Piecewise Population Growth Model I: Qualitative Exploration and Study Cases on Continuous-Time Dynamics
}

\author{
Héctor A. Echavarria-Heras $\mathbb{D}^{1},{ }^{1}$ Cecilia Leal-Ramírez, ${ }^{1}$ Guillermo Gómez, ${ }^{2}$ \\ and Elia Montiel-Arzate ${ }^{3}$ \\ ${ }^{1}$ Departamento de Ecología, Centro de Investigación Científica y de Estudios Superiores de Ensenada, \\ Carretera Ensenada-Tijuana No. 3918 Zona Playitas CP, Ensenada 22860, BC, Mexico \\ ${ }^{2}$ Facultad de Ciencias, Universidad Nacional Autónoma de México, Avenida Universidad 3000 Circuito Exterior S/N, \\ Delegación Coyoacán, CP Ciudad Universitaria, D.F.México 04510, Mexico \\ ${ }^{3}$ Instituto Tecnológico de México, Boulevard Tecnológico \#150, Ex Ejido Chapultepec, C.P, Ensenada 22780, BC, Mexico \\ Correspondence should be addressed to Héctor A. Echavarria-Heras; heheras@icloud.com
}

Received 13 August 2021; Accepted 8 October 2021; Published 27 December 2021

Academic Editor: Jorge-Antonio Lopez-Renteria

Copyright (c) 2021 Héctor A. Echavarria-Heras et al. This is an open access article distributed under the Creative Commons Attribution License, which permits unrestricted use, distribution, and reproduction in any medium, provided the original work is properly cited.

\begin{abstract}
We examine the comportment of the global trajectory of a piecewisely conceived single species population growth model. Formulation relies on what we develop as the principle of limiting factors for population growth, adapted from the law of the minimum of Liebig and the law of the tolerance of Shelford. The ensuing paradigm sets natality and mortality rates to express through extreme values of population growth determining factor. Dynamics through time occur over different growth phases. Transition points are interpreted as thresholds of viability, starvation, and intraspecific competition. In this delivery, we focus on the qualitative study of the global trajectory expressed on continuous time and on exploring the feasibility of analytical results against data on populations growing under experimental or natural conditions. All study cases sustained fittings of high reproducibility both at empirical and interpretative slants. Possible phase configurations include regimes with multiple stable equilibria, sigmoidal growth, extinction, or stationarity. Here, we also outline that the associating discrete-time piecewise model composes the logistic map applied over a particular region of the phase configuration. Preliminary exploratory analysis suggests that the logistic map's chaos onset could surpass once the orbit enters a contiguous phase region.
\end{abstract}

\section{Introduction}

As a rule, mathematical models that attempt to describe the dynamics of two or more populations, subject to a specific type of interaction in an ecosystem, are formulated based on too rigid hypotheses, resulting in poor concordance of the model's predictions with the natural process. The high degree of complexity of biological phenomena and their significant spatial-temporal variations do not assimilate by single-rule models, which are incapable of presenting the functional diversity required by levels of reliable prediction. However, when trying to include more biological information in the model hypotheses, it is often possible to fall into the opposite situation: creating models or techniques so complicated that they are also inoperative from an analytical standpoint.

From the traditional point of view, in building a model, it is necessary to include the most representative processes of the system that we are trying to describe to maintain simplicity as much as possible without losing relevant information. The problem reduces to a quest for those key processes that govern the interaction dynamics we are interested in studying. For example, in demographic ecology, the dynamics of a population depend on the correlation 
between opposing influences such as birth generating and growth inhibitory processes. Each of these effects depends on a series of factors inherent to population growth. For example, births depend on age composition, sex proportion, fertility, available food, and so on. Correspondingly, the growth inhibition process may be set by population density, disease, abiotic factors such as temperature, humidity, pollution, and others. As the number of determining factors increases, the model's complexity affects tractability and interpretability. An alternative approach is considering a horizontal integration of complexity through a piecewise modeling strategy. While embracing this approach to gain interpretative strength, it would be desirable to imbue the construct of a mechanistic profile. This work explains how we build a piecewise population model (PPM) by following a systematic way, that is, creating a piecewise description of population growth by relying upon a logical deductive approach. An ad hoc logical system that could sustain such an enterprise is what we comprehend as a principle of limiting factors (PLF) for population growth. This paradigm entailed the derivation of a PPM as a collection of submodels called growth phases that continuously compose the global trend of population size. Each growth phase describes the dynamics over a specific time interval. The naming composite further refers to the principle of limiting factors-driven piecewise population model, which also represents utilizing the PLFPPM acronym.

As we elucidate in what follows, the PLF-PPM can be arranged by following ideas in [1-3]. Mainly, the construct presented here reviews the approach in [3] to consider a modification taking into account the abatement effect that the population induces on an external resource necessary to guarantee its growth and permanence. The present PLFPPM formulation also includes a specific scaling or weighting of population size to model the increase in mortality promoted by low population densities [4]. The resulting device allows meaningful ecological interpretation of consequential growth phases. Mainly for keeping this paper's extension manageable, we focus on the continuoustime form of the PLF-PPM. Nevertheless, we judged that it is pertinent to advance an outline of the next discrete-time version, whose detailed exploration will be addressed in the second part of this work. We include several study cases that show the empirical and interpretative adequacy of the present paradigm. The Appendix presents a qualitative study of associating continuous-time global trajectory.

\section{Materials and Methods}

2.1. The General Piecewise Population Growth Model Setup. Throughout this paper, symbol $R$ stands for the set of real numbers and $R^{+}$denotes the subset of $R$ whose elements are positive. Correspondingly, the size of a population at a positive time $t$ represents employing $x(t)$ and formally stands as a function having domain $R^{+}$and range $X_{R}$, a proper subset of $R^{+}$, that is, $x(t): R^{+} \longrightarrow X_{R} \subseteq R^{+}$. For the case of an isolated population, a customary assumption establishes the existence of a function $G(x): X_{R} \longrightarrow R$ such that

$$
\dot{x}(t)=G(x(t)) \cdot x(t)
$$

where $\dot{x}(t)$ stands for the rate of change of the number $x(t)$ of individuals in the population at time $t$. The function $G(x)$ is interpreted as an average estimate of the influence of all the processes that govern population growth. More specifically, this function embodies the average growth rate for each individual in the population. In the following, we will refer to $G(t)$ as the per capita growth rate or the intrinsic growth rate of $x(t)$.

Determining the function $G(x)$ in a closed form is usually impossible or very complicated. However, from an empirical perspective, it is possible to suppose that the function $G(x)$ admits expansion as a power series, with which we would obtain

$$
G(x)=\sum_{i=0}^{\infty} a_{i} x^{i} .
$$

Then, for example, neglecting the $a_{n} x^{n}$ terms with $n \geq 2$, we could consider the approximation

$$
G(x)=r-k x
$$

The above characterization of the intrinsic growth rate $G(x)$ establishes the form of the differential equation (1) that leads to the identification of the so-called logistic curve, commonly adopted as a model of population growth in a limited environment. Although it is feasible to give a biological interpretation to equation (3) model, it shows the fragility inherent in all simplified models, which mainly relates to the lack of suitable complexity. Moreover, the variation of population size could compose an outstanding array of patterns. Then, maintaining a single association rule for $G(x)$, through the full extension of $X_{R}$, no matter how complex such a rule conceives, it could render insufficient to imbue a consistent reproducibility of observed population values. A traditional approach to adapt $G(x)$ is by considering a polynomial approximation of degree $n$ derived from the infinite series of equation (2). Nevertheless, this approach bears a disadvantage since at gaining reproducibility strength, we could lose interpretability.

Before getting into matters on suitable complexity embedding of $G(x)$, we introduce some notation conventions. Given a function $S(t): A \longrightarrow B$ and a subset $C \subseteq A$, the restriction of $S(t)$ to the set, $C$ denotes $\left.S(t)\right|_{C}: C \longrightarrow B$, it is formally defined by

$$
\left.S(t)\right|_{C}=S(t) \text { for each } t \text { in C. }
$$

A second method of adapting the complexity attending to an optimal reproducibility criterion is conceiving $G(x)$ as a composite of $m$ operating modes, each one characterized by a continuous and differentiable function $G_{i}(x): \mathfrak{R}_{i}[x] \longrightarrow R$ being $\mathfrak{R}_{i}[x]$ a region defined through

$$
\mathfrak{R}_{i}[x]=\left\{x \mid p_{i-1}<x \leq p_{i}\right\},
$$

for $i=1,2, \ldots, m$. The components of the vector $\mathbf{P}=\left(p_{0}, \ldots, p_{m}\right)$ represent breakpoints for transition among the operating modes $G_{i}(x)$. We also assume that the 
collection of regions $\Re_{i}[x]$ provides a covering for the range of $x(t)$, that is, $X_{R} \subseteq \cup_{1}^{m}\left(\Re_{i}[x]\right)$. We could formally set

$$
G_{i}(x)=\left.G(x)\right|_{\Re_{i}[x]} .
$$

So, we can represent $G(x)$ expanded by the $G_{i}(x)$ submodels for $i=1,2, \ldots, m$, namely,

$$
G(x)=\sum_{i=1}^{m} \chi\left(\Re_{i}[x]\right) G_{i}(x),
$$

with $\chi\left(\Re_{i}[x]\right)$ representing the characteristic function of $\mathfrak{R}_{i}[x]$, namely,

$$
\chi\left(\Re_{i}[x]\right)= \begin{cases}1, & \text { if } x(t) \in \mathfrak{R}_{i}[x], \\ 0, & \text { if } x(t) \in \mathfrak{R}_{i}[x]^{c},\end{cases}
$$

Equation (7) intends to provide a piecewise description of the intrinsic population growth rate in the autonomous differential equation (1). Discretizing the resulting differential equation, we can identify the parameters defining the $G_{i}(x)$ submodels describing the phases of population growth, as well as the threshold parameters $p_{0}, p_{1}, \ldots, p_{m}$ that represent the transition points between the growth phases $G_{i}(x)$; given assumed continuity properties of $G(x)$, the phase changes must be set as continuous transitions between the submodels, that is, we have

$$
G_{i}\left(p_{i}\right)=\lim _{x \longrightarrow p_{i}^{-}} G_{i+1}(x), \text { for } i=1,2, \ldots, m-1 .
$$

But, in the general settings, the transition between phases at breakpoints $p_{0}, p_{1}, \ldots, p_{m}$ can occur in a discontinuous way.

In the settings of the model of equation (1), the function $f(x)=G(x) x$ is interpreted as the natural growth rate of the population. Then, defining $f_{i}(x)=G_{i}(x) x$ for $i=1,2, \ldots, m$ and keeping the notation convention entailing the associated domain regions, according to equation (6), we set

$$
f_{i}(x)=\left.f(x)\right|_{\Re_{i}[x]} .
$$

This way, we can propose a piecewise expanded form of equation (1), namely,

$$
\dot{x}(t)=\sum_{i=1}^{m} \chi\left(\Re_{i}[x]\right) f_{i}(x), \text { for } x(0)=x_{0},
$$

where $\mathfrak{R}_{i}[x]$ and $\chi\left(\mathfrak{R}_{i}[x]\right)$ are defined by equations (5) and (8) one to one.

The non-autonomous form of the dynamical system of equation (11) addresses similarly by replacing $f(x)$ by a continuous and differentiable function $f(x, t): X_{R} \times R \longrightarrow R$ and interprets $x(t)$ and $t$ as a forced form of the natural growth rate of the population. The extension of equation (11) to the non-autonomous case $f(x, t)$ follows by setting submodel $f_{i}(x, t): \Re_{i}[x, t] \longrightarrow R$ being $\mathfrak{R}_{i}[x, t]=\mathfrak{R}_{i}[x] \times R$ for $i=1,2, \ldots, m$. This way, a piecewisely expanded non-autonomous form of equation (11) could be expressed by

$$
\dot{x}(t)=\sum_{1=1}^{m} \chi\left(\Re_{i}[x, t]\right) f_{i}(x, t), \text { with } x(0)=x_{0},
$$

where $\chi\left(\mathfrak{R}_{i}[x, t]\right)$ symbolizes the characteristic function of the rectangle $\mathfrak{R}_{i}[x, t]$. Nevertheless, in this paper, we focus on the autonomous case.

To provide a piecewise representation of the solution $x(t)$ to the dynamical system of equation (11), we conceive trajectory sectors $x_{i}(t)$ for $i=1,2, \ldots, m$ defined by

$$
x_{i}(t)=\left.x(t)\right|_{T_{i}(t)} .
$$

For $T_{i}(t) \subseteq R^{+}$, the interval $T_{i}(t)=x^{-1}\left(\Re_{i}[x]\right)$. Since $x(t)$ varies continuously, we must set the consistency condition

$$
x_{i}\left(t_{i}\right)=\lim _{t \longrightarrow t_{i}^{-}} x_{i+1}(t),
$$

for $t_{i}=x^{-1}\left(p_{i}\right)$ being $p_{i}$ the breakpoint for the transition from $f_{i}(x)$ to $f_{i+1}(x)$. Then, we arrange

$$
x(t)=\sum_{i=1}^{m} \chi\left(T_{i}(t)\right) x_{i}(t), \text { for } x(0)=x_{0},
$$

with $\chi\left(T_{i}(t)\right)$ being defined by

$$
\chi\left(T_{i}(t)\right)= \begin{cases}1, & \text { if } t \in T_{i}(t), \\ 0, & \text { if } t \in T_{i}(t)^{c} .\end{cases}
$$

In any event, usually assembling equation (11) relies on an empirical description that abides by the highest reproducibility criterion. Regularly, such an approach disregards any phenomenological explanation of population growth. In this work, we explain a systematic way to obtain a collection of submodels that mimic the global trend of population size $x(t)$, where each one of them describes the dynamics over a specific time interval, that is, we build a piecewise description of both $\dot{x}(t)$ and $x(t)$ as given by equations (11) and (13) one to one by relying on a logical deductive approach. This can be obtained by following [1-3]. The corresponding derivation is based on the use of the ecological principle of limiting factors. The construct presented here corresponds to a modification of the slant in [3]. To consider the abatement effect that the population induces on an external resource necessary to guarantee its growth and permanence, the present formulation also includes a specific scaling or weighting of population size to model the increase in mortality promoted by low population densities [4].

2.2. The Principle of Limiting Factors. German Physiologist Justus Von Liebig [5], studying the growth of certain plants, realized that to guarantee their development, there had to be a set of essential nutrients. Some had to be abundant, and others were required only in small quantities. A significant discovery of Liebig was that the absence of some nutrient could not be replaced with any other that appeared in abundance. Moreover, a medium that contained all the nutrients in abundance except one of them, which appeared in insufficient quantity, would allow plant growth until the 
nutrient was utterly depleted. The absence of some element limited growth. In addition, when growth occurred, the latter turned out to be controlled by the nutrient appearing in lesser proportion. Liebig called this regularity the law of the minimum. Later, in [6], the North American Ecologist V. E. Shelford extended the domain of Liebig's law to what is now known as the law of tolerance. Shelford pointed out that when there is an excess of a specific element, this can be as limiting as its deficiency. It follows that all the processes that determine the dynamics of a population will occur in intensities governed by the minima or maxima of the factors that nourish these processes. We refer to this notion as the principle of limiting factors (PLF) for simplicity and following the tradition. It intends to interpret the law that limits a process through the maximum and minimum of factors acting in the analyzed system.

2.3. The Principle of Limiting Factors-Driven Piecewise Population Growth Model. Considering the validity of the principle of limiting factors, let us construct a mathematical model to interpret the performance of a population whose size at time $t$ is denoted by $x(t)$. We suppose that departing from a value $x(0)=x_{0}$, the population growth depends on an external resource whose availability at time $t$ is modeled by a continuous and differentiable function $R(t)$ with rank in $R^{+}$. We will also assume that the natural growth rate of the population size $\dot{x}(t)$ as given by equation (2) expresses through a function $f(x)=G(x) x$ that determines on a first instance by the intensities of two opposing processes, one of birth $N(x)$ yielding offspring and another $M(x)$ determining the death of the individuals, that is,

$$
f(x)=\beta N(x)-\mu M(x)
$$

where $\beta$ and $\mu$ are positive scaling parameters [2, 3], $N(x)$ stands for the strength of the process leading to increasing population size, that is, the rate at which the number of newborn in the population is generated, and $M(x)$ represents the intensity of the growth-inhibiting process, that is, the mortality rate. We assume that $N(x)$ and $M(x)$ both express through continuous and differentiable functions.

Then, according to the principle of limiting factors, we assume that $N(x)$ depends essentially on two factors: one being the size of the population $x(t)$ and the second one taken as the amount of feeding energy available; this symbolized employing $R(x)$. We suppose that before the population began exhausting the resource $R(x)$, this is kept at a constant value $F_{0}$. Additionally, we think through that the total weight of the population expresses in the same units of energy as $R(x)$. Let us also undertake that each individual in the $x(t)$ pool maintains an energy consumption equal to a proportion $k$ of its weight. Then, we can assume in the first instance that $R(x)$ represents using the linear model

$$
R(x)=F_{0}-k x(t) .
$$

Then, resource availability $R\left(x_{o}\right)$ will plentifully suit feeding requirements of the initial population $x_{o}$ whenever the condition $R\left(x_{o}\right) \gg k x_{o}$ satisfies or equivalently according to equation (18) whenever $F_{0} \gg 2 k x_{0}$ holds. We will then have that if the amount of energy consumed by the population at time $t$, i.e., $k x(t)$, is less than $R(x)$, there will be enough food for all individuals, that is, the availability of energy will guarantee that the population can participate in the reproduction process unrestrictedly, so we can establish the assumption that the birth rate will be proportional to the size of the population, that is, we can set the equality $N(x)=x(t)$. In the case in which $k x(t)$ exceeds $R(x)$, then only as many individuals of the population energetically equivalent to $R(x)$ will be able to feed. Therefore, the reproductive potential of the population will be limited. That is, according to Liebig's principle, energy availability will be a limiting factor for population growth. Then, $N(x)=R(x)$. Thus, we can define $N(x)$ by

$$
N(x)=\min _{t}\{k x(t), R(x)\} .
$$

Solving the inequality $k x(t)<F_{0}-k x(t)$, we can express equation (19) in the equivalent form

$$
N(x)=\min _{t}\{x(t), E\}
$$

where the constant $E=F_{0} / 2 k$ is interpreted as a starvation threshold since if $x(t)$ grows maintaining levels higher than $E$, the number of offspring will turn to be controlled by the external factor $F_{0}$ and the rate of individual energy consumption $k$ that could eventually lead to the decline of the population.

Concerning the intensity of the natural death process $M(x)$, we will consider this, considering Shelford's law of tolerance based on population density as the critical factor. According to Allee's principle, reduced population levels will restrict the number of interactions between individuals, making mating difficult, implying decreasing recruitment, favoring even a lower number of individuals, and eventually leading to the total depletion of the population. In this circumstance, we will say that the strength of the mortality process will be proportional to the size of the population, that is, $M(x)=a x(t)$, where $a$ is a positive constant. On the other hand, at high population sizes, density upsurges, the struggle for resources intensifies, and so does the transmission of diseases, thereby increasing mortality. In this case, we take the intensity of the mortality process as being proportional to the number of encounters between the individuals, that is, $M(x)=b x^{2}(t)$ where $b \in R^{+}$. Then, according to Shelford's law of tolerance, we can express $M(x)$ in the form

$$
M(x)=\max _{t}\left\{a x(t), b x^{2}(t)\right\} .
$$

In summary, the PLF and equations (11) and (17) imply the piecewise continuous form

$$
\dot{x}(t)=\sum_{i=1}^{m} \chi\left(\Re_{i}[x]\right) f_{i}(x), \text { for } x(0)=x_{0},
$$

where $f_{i}(x)$ and $\mathfrak{R}_{i}[x] i=1,2,3,4$ are given by 


$$
\begin{array}{ll}
f_{1}(x)=\beta E-\mu b x(t)^{2} & \mathfrak{R}_{1}[x]=\left\{x(t) \mid(x(t)>E) \wedge\left(x(t)>\frac{a}{b}\right)\right\} \\
f_{2}(x)=\beta x(t)-\mu b x(t)^{2} & \mathfrak{R}_{2}[x]=\left\{x(t) \mid(x(t)<E) \wedge\left(x(t)>\frac{a}{b}\right)\right\} \\
f_{3}(x)=(\beta-\mu a) x(t) & \mathfrak{R}_{3}[x]=\left\{x(t) \mid(x(t)<E) \wedge\left(x(t)<\frac{a}{b}\right)\right\} \\
f_{4}(x)=\beta E-\mu a x(t) & \mathfrak{R}_{4}[x]=\left\{x(t) \mid(x(t)>E) \wedge\left(x(t)<\frac{a}{b}\right)\right\}
\end{array}
$$

with $\chi\left(\mathfrak{R}_{i}[x]\right)$ representing the characteristic function of $\mathfrak{R}_{i}[x]$ for $i=1,2,3,4$ (cf. equation (8)). In what follows, the principle of limiting factors-driven piecewise population growth model of equations (22) and (23) will be referred to as PLF-PPM for short.

Since $x(t)$ stands for the solution to equation (22), then according to equation (15), we have that $x_{i}(t)=\left.x(t)\right|_{T_{i}(t)}$ for $i=1,2,3,4$, stands for the $i$ th sector of the PLF driven trajectory $x(t)$ having range in $\Re_{i}[x]$, and correspondingly, this sets $T_{i}(t)=x^{-1}\left(\Re_{i}[x]\right)$. Then, $x_{i}(t)$ solves the differential equation $\dot{x}_{i}(t)=f_{i}(x(t))$ with $x_{i}(t)$ satisfying $x_{i}(0)=$ $x_{i 0} \in \mathfrak{R}_{i}[x]$ and the restriction $t \in T_{i}(t)$. Therefore, we have

$$
\begin{aligned}
& x_{1}(t)=\frac{\lambda_{1}\left(C-e^{-2 t \sqrt{\beta \mu b E}}\right)}{C+e^{-2 t \sqrt{\beta \mu b E}}} \quad x(t) \in \mathfrak{R}_{1}[x], t \in T_{1}(t) \quad x_{10}=\frac{\lambda_{1}(C-1)}{C+1} \quad \lambda_{1}=\sqrt{\frac{\beta E}{\mu b}} \\
& x_{2}(t)=\frac{C \lambda_{2}}{\lambda_{2} e^{-\beta t}+C} \\
& x(t) \in \mathfrak{R}_{2}[x], t \in T_{2}(t) \quad x_{20}=\frac{\beta C}{\beta+\mu b C} \quad \lambda_{2}=\frac{\beta}{\mu b} \\
& x_{3}(t)=C e^{(\beta-\mu a) t} \\
& x(t) \in \mathfrak{R}_{3}[x], t \in T_{3}(t) \quad x_{30}=C \\
& x_{4}(t)=C e^{-\mu a t}+\lambda_{4}\left(1-e^{-\mu a t}\right) \\
& x(t) \in \mathfrak{R}_{4}[x], t \in T_{4}(t) \\
& x_{40}=C \\
& \lambda_{4}=\frac{\beta E}{\mu a}
\end{aligned}
$$

with $\lambda_{i}$ being the equilibrium solutions as determined by the natural growth rates $f_{i}(x(t))$ (cf. equation (24)). Additionally, the submodels $x_{i}(t)$ composing the solution $x(t)$ as given by equation (15) determine by the order relationship that $E$ and $a / b$ satisfy. Accordingly, the global trajectory $x(t)$ could fit to three possible topologies, one type $M_{1}$ associating to the statement $M_{1}:(E>a / b)$, another form $M_{2}$ consistent to $M_{2}:(E>a / b)$; and a third form $M_{3}$ associating to $M_{3}:(E>a / b)$ the phase composite $P_{1}(t, x(t))$ for $M_{1}$ models will include regions $\mathfrak{R}_{1}[x], \mathfrak{R}_{2}[x], \quad$ and $\mathfrak{R}_{3}[x], \quad$ that is, $P_{1}(t, x(t))=\cup_{i \neq 4}\left\{\Re_{i}[x]\right\} \bigcup(0, \infty)$. Correspondingly, for models $M_{2}$, phase arrangement becomes $P_{2}(t, x(t))=\cup_{i \neq 2}\left\{\mathfrak{R}_{i}[x]\right\} \bigcup(0, \infty)$. In turn, phase portrait for models $M_{2}$ and the regions $\Re_{2}[x]$ and $\Re_{4}[x]$ is mutually exclusive. For models $M_{3}$, the phase combination turns to $P_{3}(t, x(t))=\cup_{i \neq 2,4}\left\{\Re_{i}[x]\right\} \bigcup(0, \infty)$. The Appendix presents a qualitative study of trajectories resulting from equation (22).

\section{Results}

3.1. Analytic Exploration. To study the varied forms of the global trajectory $x(t)$ derived from the PLF-PM, we begin by setting the array of possible phase configurations associated with the basic parameters $a, b, E, \beta$, and $\mu$ (cf. equations (22) and (23)). We depart from the ordering relationship for the external energy $E$ and the ratio of mortality from population density proportions, $a / b$, that yields a first model type classification $\left(M_{i}, i=1,2,3\right)$. We have to consider models $M_{1}$ associated to the inequality $E>a / b$, where the range of $x(t)$ composes the regions: $\mathfrak{R}_{1}[x]=\{a / b<E<x\}$, $\mathfrak{R}_{2}[x]=\{a / b<x<E\}$, and $\mathfrak{R}_{3}[x]=\{x<a / b<E\} \quad$ (cf. equation (23)). For model type $M_{2}$ where the relationship $E>a / b$ holds, the range of $x(t)$ will be divided into the regions $\mathfrak{R}_{1}[x], \mathfrak{R}_{3}[x]$, and $\mathfrak{R}_{4}[x]=\{x<a / b<E\} \quad$ (cf. equation (23)). For model type $M_{3}$ linking to the ordering $E=a / b$, the range of $x(t)$ will be divided into the regions $\mathfrak{R}_{1}[x]$ and $\mathfrak{R}_{3}[x]$. We can be aware that regions $\mathfrak{R}_{2}[x]$ and 
$\mathfrak{R}_{4}[x]$ are mutually excluding. Besides, it also requires classifying possible phase arrangements according to a Birth to Mortality Scaled Ordering $\left(\mathrm{BMS}_{i}, i=1,2,3\right)$, namely, $\mathrm{BMS}_{1}$ : when $(\beta<\mu a), \mathrm{BMS}_{2}$ : if $(\beta>\mu a)$, and $\mathrm{BMS}_{3}$ : whenever $(\beta=\mu a)$. Additionally, each model type $M_{i}$ with $i=1,2$ determines possible placements of the initial condition, and each one associated to composing phase regions $\mathfrak{R}_{i}[x]$ with $i=1,2,3,4$ determines an initial condition ordering $\left(\mathrm{IC}_{i k(i)}\right.$ with $i=1,2$ and $\left.k(i)=1,2, \ldots, 5\right)$, namely, $\mathrm{IC}_{11}$ : for $\left(E<x_{o}\right), \mathrm{IC}_{12}$ : whenever $\left(a / b<x_{o}<E\right), \mathrm{IC}_{13}$ : when $\left(x_{o}<a / b\right), \mathrm{IC}_{14}$ : if $\left(x_{o}=E\right)$, and $\mathrm{IC}_{15}$ : whenever $\left(x_{o}=a / b\right)$. Correspondingly, $\mathrm{IC}_{21}$ : for $\left(x_{0}<E\right), \quad \mathrm{IC}_{22}$ : whenever $\left(E<x_{0}<a / b\right), \mathrm{IC}_{23}$ : when $\left(x_{o}>a / b\right), \mathrm{IC}_{24}$ : if $\left(x_{o}=E\right)$, and $\mathrm{IC}_{25}$ : whenever $\left(x_{o}>a / b\right)$. The possible phase arrangements typify according to a three-dimensional conjunction operator $\mathscr{H}[\mathbf{i}, \mathbf{j}, \mathbf{k}(\mathbf{i})]=\left(M_{i} \wedge \mathrm{BMS}_{j} \wedge \mathrm{IC}_{i k(i)}\right)$ for $i$ and $k(i)$ as specified. Table 1 summarizes phase arrangements for model type $M_{1}$, and Table 2 summarizes those corresponding to model type $M_{2}$. In addition to the $\mathscr{H}[\mathbf{i}, \mathbf{j}, \mathbf{k}(\mathbf{i})]$ conjunctions, we must consider the positioning of the equilibrium solutions inside their associated regions. For this reason, in the presentation of results, we could use the $O \lambda(i, j)$ indicator for $i=1,2,4$ designating equilibrium $\lambda_{i}$ and $j=1,2,4$ describing region $\Re_{i}[x]$. Then, for instance, $O \lambda(1,1)$ identifies the placement of equilibrium $\lambda_{1}$ inside region $\Re_{1}[x]$; correspondingly, $O \lambda(2,3)$ labels positioning of $\lambda_{2}$ inside region $\mathfrak{R}_{3}[x]$ and so on. Yet given $O \lambda(i, j)$, this relates to distinguishing how the initial condition is positioned relative to the equilibrium solution $\lambda_{i}$. Resulting orderings generically symbolize through $\operatorname{IC} \lambda(i, j)$ for $i=1,2,4$ associated to the equilibrium solution $\lambda_{i}$ and $j=1,2$ labeling the ordering of $x_{0}$ relative to $\lambda_{i}$. This way, $\operatorname{IC} \lambda(i, 1)$ stands for $\left(x_{0}<\lambda_{i}\right)$ and IC $\lambda(i, 2)$ stands for $\left(x_{0}>\lambda_{i}\right)$. Besides described symbols, we could refer to direct inequalities that a given equilibrium satisfies relative to thresholds defining regions.

Then, for instance, the statement $(\mathscr{H}[2,2,1] \wedge \operatorname{IC} \lambda(i, j) \wedge \operatorname{IC\lambda }(1,1)) \equiv 1$ signifies that we have a model type $M_{1}$ with $\beta>\mu a$ and that the initial condition satisfies $a / b<x_{o}$, the $\lambda_{1}$ equilibrium in region $\Re_{1}[x]$, that is, $\lambda_{1}>a / b$, and finally that $a / b<x_{o}<\lambda_{1}$. In the Appendix, we present an extended qualitative study of the performance of the global trajectory $x(t)$ under conjunctions $\mathscr{H}[\mathbf{i}, \mathbf{j}, \mathbf{k}(\mathbf{i})] \wedge O \lambda(i, j) \wedge \operatorname{IC\lambda }(i, j)$.

Figure 1(a) shows the performance of the PLF-PM composite trajectory $x(t)$ for the $\mathscr{H}[\mathbf{1}, \mathbf{1}, \mathbf{1}]$ configuration. Particularly, shown placement $a / b<\lambda_{1}<E$ derives from the auxiliary ordering $E>\mu a^{2} / \beta b$. The global trajectory $x(t)$ initiates in a region $\mathfrak{R}_{1}[x]$ according to the $x_{1}(t)$ rule. Then, since for $\beta<\mu a$ population size decreases, continuity of linked trajectory projects a time $t_{1}$ such that $x(t)$ reaches the $E$ threshold, that is, $x\left(t_{1}\right)=E$, and then $x(t)$ enters into the region $\Re_{2}[x]$ switching to the $x_{2}(t)$ rule. The settings imply the existence of a second time $t_{2}$ so that $x(t)$ touches the $a / b$ frontier, thereby getting into the region $\Re_{3}[x]$ and following according to the $x_{3}(t)$ rule. But, since $\beta<\mu a$, eventually $x(t)$ vanishes. $\mathscr{H}[1,1,2]$ and $\mathscr{H}[1,1,3]$ configurations similarly tied to the $\beta<\mu a$ ordering also drive population size to vanish Figure 1(b) pertains to the $\mathscr{H}[2, \mathbf{1}, \mathbf{1}]$ arrangement. Composite trajectory $x(t)$ begins in the region $\mathfrak{R}_{1}[x]$
TABle 1: Basic phase arrangements $\mathscr{H}[1, \mathbf{j}, \mathbf{k}(\mathbf{i})]$ determined by the PLF-PM and phase arrangements for model type $M_{1}$ associated to the $E>a / b$ ordering.

\begin{tabular}{lcllcc}
\hline $\mathscr{H}[i, j, k(i)]$ & $M_{1}$ & $\mathrm{BMS}_{i}$ & $\mathrm{BMS}_{i}$ & $I C_{i k(i)}$ & $I C_{i k(i)}$ \\
\hline $\mathscr{H}[1,1,1]$ & $E>a / b$ & $\mathrm{BMS}_{1}$ & $\beta<\mu a$ & $\mathrm{IC}_{11}$ & $E<x_{o}$ \\
$\mathscr{H}[1,1,2]$ & $E>a / b$ & $\mathrm{BMS}_{1}$ & $\beta<\mu a$ & $\mathrm{IC}_{12}$ & $a / b<x_{o}<E$ \\
$\mathscr{H}[1,1,3]$ & $E>a / b$ & $\mathrm{BMS}_{1}$ & $\beta<\mu a$ & $\mathrm{IC}_{13}$ & $x_{o}<a / b<E$ \\
$\mathscr{H}[1,1,4]$ & $E>a / b$ & $\mathrm{BMS}_{1}$ & $\beta<\mu a$ & $\mathrm{IC}_{14}$ & $x_{o}=E$ \\
$\mathscr{H}[1,1,5]$ & $E>a / b$ & $\mathrm{BMS}_{1}$ & $\beta<\mu a$ & $\mathrm{IC}_{15}$ & $x_{o}=a / b$ \\
$\mathscr{H}[1,2,1]$ & $E>a / b$ & $\mathrm{BMS}_{2}$ & $\beta>\mu a$ & $\mathrm{IC}_{21}$ & $E<x_{o}$ \\
$\mathscr{H}[1,2,2]$ & $E>a / b$ & $\mathrm{BMS}_{2}$ & $\beta>\mu a$ & $C_{22}$ & $a / b<x_{o}<E$ \\
$\mathscr{H}[1,2,3]$ & $E>a / b$ & $\mathrm{BMS}_{2}$ & $\beta>\mu a$ & $\mathrm{IC}_{23}$ & $x_{o}<a / b<E$ \\
$\mathscr{H}[1,2,4]$ & $E>a / b$ & $\mathrm{BMS}_{2}$ & $\beta>\mu a$ & $\mathrm{IC}_{24}$ & $x_{o}=E$ \\
$\mathscr{H}[2,2,5]$ & $E>a / b$ & $\mathrm{BMS}_{2}$ & $\beta>\mu a$ & $\mathrm{IC}_{25}$ & $x_{o}=a / b$ \\
\hline $\mathscr{H}$
\end{tabular}

$j=1,2$ sign discriminates ordering between $\beta$ and $\mu a$, and the composing index $k(i)$ differentiates the position of the initial condition among the phase regions constituting a model type $M_{1}$.

according to the $x_{1}(t)$ rule, but $\beta<\mu a$, and then population size decreases, so at a time $t_{1}$, it reaches the $a / b$ threshold, that is, $x\left(t_{1}\right)=a / b$, entering into the region $\mathfrak{R}_{4}[x]$ and subsequently abiding by the rule $x_{4}(t)$. The decreasing trend implies population size touching the $E$ boundary at a second time $t_{2}$, thereby placing within the region $\mathfrak{R}_{3}[x]$ and then switching again, this time to the $x_{4}(t)$ rule. Afterward, population size keeps decreasing following an asymptotic trend to extinction. The faith of the PLF-PM composite trajectory $x(t)$ for $\mathscr{H}[\mathbf{2}, \mathbf{1}, \mathbf{2}]$ and $\mathscr{H}[\mathbf{2}, \mathbf{1}, \mathbf{3}]$ is extinction equally. Figure 1 (c) pertains to the $\mathscr{H}[3,1,1]$ conjunction corresponding to the $x_{o}>E$ ordering. Then, in the beginning, population size $x(t)$ places in the region $\Re_{1}[x]$ and decreases according to the $x_{1}(t)$ rule. Subsequently, it asymptotically approaches the value $\lambda_{1}<E$, but on its trend, it hits the $E$ boundary at time $t_{1}$ switching to rule $x_{3}(t)$ holding in region $\Re_{3}[x]$. The condition $\beta<\mu a$ drives population to extinction. For $\mathscr{H}[3,1,2]$ conjunction, the initial condition placement $x(0)<E$ keeps the population size within region $\mathfrak{R}_{3}[x]$ where it progresses to extinction according to the $x_{3}(t)$ trajectory.

Assume $(O \lambda(2,1) \wedge \mathscr{H}[\mathbf{1}, \mathbf{2}, \mathbf{1}]) \equiv 1$; then, the condition $\mathrm{BMS}_{1}$ : $(\beta<\mu a)$ holds; additionally, the $\lambda_{2}$ equilibrium localizes above $E$, that is, $O \lambda(2,1):\left(\lambda_{2}>E\right)$, and these orderings appear in conjunction with $M_{1}:(E>a / b)$ and $\mathrm{IC}_{11}:\left(E<x_{o}\right)$. Particularly, $\lambda_{2}>E$ implies $\lambda_{1}>E$ so the initial condition placement $E<x_{o}<\lambda_{1}$ sets the $x(t)$ trajectory to increase according to the $x_{1}(t)$ rule, approaching the asymptotic limit $\lambda_{1}$ and staying within region $\Re_{1}[x]$. On the other hand, whenever we have $E<\lambda_{1}<x_{o}$, population size $x(t)$ decreases asymptotically towards $\lambda_{1}$. This establishes $\lambda_{1}$ as a stable equilibrium in region $\mathfrak{R}_{1}[x]$ (see Figure $2(\mathrm{a})$ ). Alternatively, under the statement $(O \lambda(2,2) \wedge \mathscr{H}[\mathbf{1}, \mathbf{2}, 2]) \equiv 1$, since we have $\beta>\mu a$, the ordering $\lambda_{2}>a / b$; then, since $O \lambda(2,2)$ implies $\lambda_{2}<E$, we could have $a / b<x_{o}<\lambda_{2}<E$ or $a / b<\lambda_{2}<x_{o}<E$. If $a / b<x_{o}<\lambda_{2}<E$ gets true, $x(t)$ will behave according to the $x_{2}(t)$ law, and then it will decrease from $x_{o}$ and approach the asymptotic limit $\lambda_{2}$. For $a / b<\lambda_{2}<x_{o}<E$, the population size $x(t)$ still conforming to the $x_{2}(t)$ growth law will increase from $x_{o}$ and approach $\lambda_{2}$. Thus, $\lambda_{2}$ stands for a stable 
TABLE 2: Basic phase arrangements $\mathscr{H}[2, j, k(i)]$ determined by the PLF-PM and phase arrangements for model type $M_{2}$ associated to the $E<a / b)$ ordering.

\begin{tabular}{|c|c|c|c|c|c|}
\hline $\mathscr{H}[i, j, k(i)]$ & $M_{2}$ & $\mathrm{BMS}_{i}$ & $\mathrm{BMS}_{i}$ & $\mathrm{IC}_{i k(i)}$ & $\mathrm{IC}_{i k(i)}$ \\
\hline $\mathscr{H}[2,1,1]$ & $E<a / b$ & $\mathrm{BMS}_{1}$ & $\beta<\mu a$ & $\mathrm{IC}_{11}$ & $E<x_{o}$ \\
\hline $\mathscr{H}[2,1,2]$ & $E<a / b$ & $\mathrm{BMS}_{1}$ & $\beta<\mu a$ & $\mathrm{IC}_{12}$ & $a / b<x_{o}<E$ \\
\hline $\mathscr{H}[2,1,3]$ & $E<a / b$ & $\mathrm{BMS}_{1}$ & $\beta<\mu a$ & $\mathrm{IC}_{13}$ & $x_{o}<a / b<E$ \\
\hline $\mathscr{H}[2,1,4]$ & $E<a / b$ & $\mathrm{BMS}_{1}$ & $\beta<\mu a$ & $\mathrm{IC}_{14}$ & $x_{o}=E$ \\
\hline $\mathscr{H}[2,1,5]$ & $E<a / b$ & $\mathrm{BMS}_{1}$ & $\beta<\mu a$ & $\mathrm{IC}_{15}$ & $x_{o}=a / b$ \\
\hline $\mathscr{H}[2,2,1]$ & $E<a / b$ & $\mathrm{BMS}_{2}$ & $\beta>\mu a$ & $\mathrm{IC}_{21}$ & $E<x_{o}$ \\
\hline $\mathscr{H}[2,2,2]$ & $E<a / b$ & $\mathrm{BMS}_{2}$ & $\beta>\mu a$ & $C_{22}$ & $a / b<x_{o}<E$ \\
\hline $\mathscr{H}[2,2,3]$ & $E<a / b$ & $\mathrm{BMS}_{2}$ & $\beta>\mu a$ & $\mathrm{IC}_{23}$ & $x_{o}<a / b<E$ \\
\hline $\mathscr{H}[2,2,4]$ & $E<a / b$ & $\mathrm{BMS}_{2}$ & $\beta>\mu a$ & $\mathrm{IC}_{24}$ & $x_{o}=E$ \\
\hline $\mathscr{H}[2,2,5]$ & $E<a / b$ & $\mathrm{BMS}_{2}$ & $\beta>\mu a$ & $\mathrm{IC}_{25}$ & $x_{o}=a / b$ \\
\hline
\end{tabular}

$j=1,2$ sign discriminates ordering between $\beta$ and $\mu a$, and the composing index $k(i)$ differentiates the position of the initial condition among the phase regions constituting a model type $M_{2}$

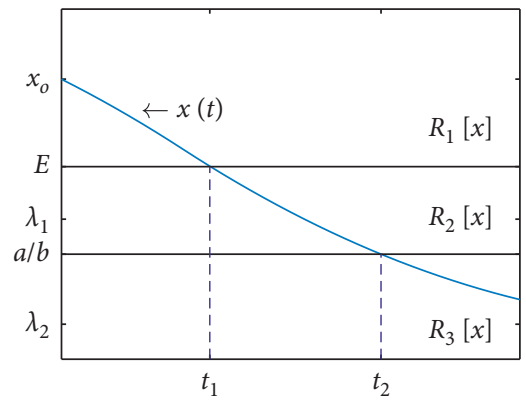

(a)

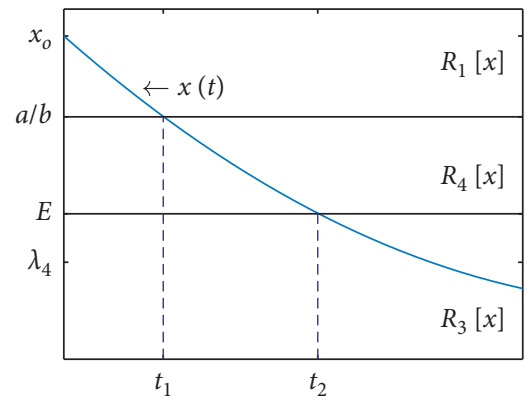

(b)

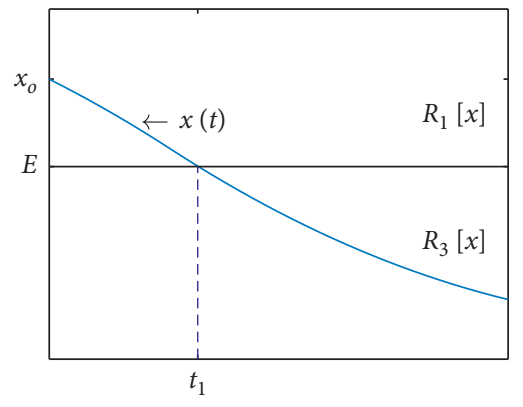

(c)

FIgURe 1: Examples of the construction of the PLF-PM composite trajectory $x(t)$ for $\mathscr{H}[\mathbf{i}, \mathbf{1}, \mathbf{1}]$ for $i=1,2,3$; these configurations include a $\mathrm{BMS}_{1}:(\beta<\mu a)$ condition. (a) Composite trajectory $x(t)$ for $\mathscr{H}[\mathbf{1}, \mathbf{1}, \mathbf{1}]$. (b) Composite trajectory $x(t)$ for $\mathscr{H}[\mathbf{2}, \mathbf{1}, \mathbf{1}]$. (c) Composite trajectory $x(t)$ for $\mathscr{H}[3,1,1]$.

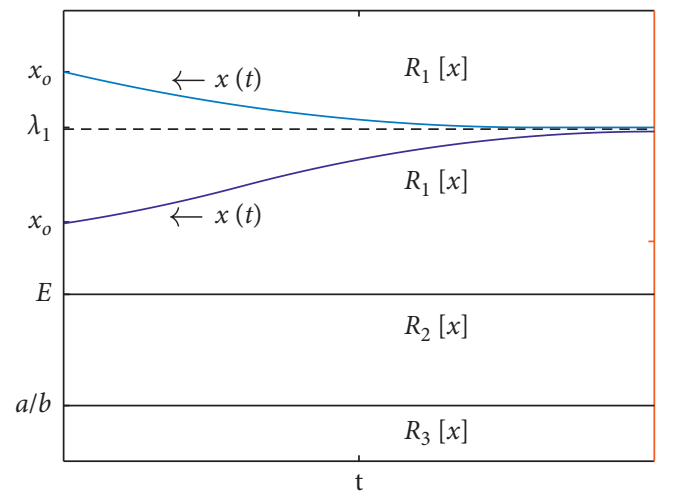

(a)

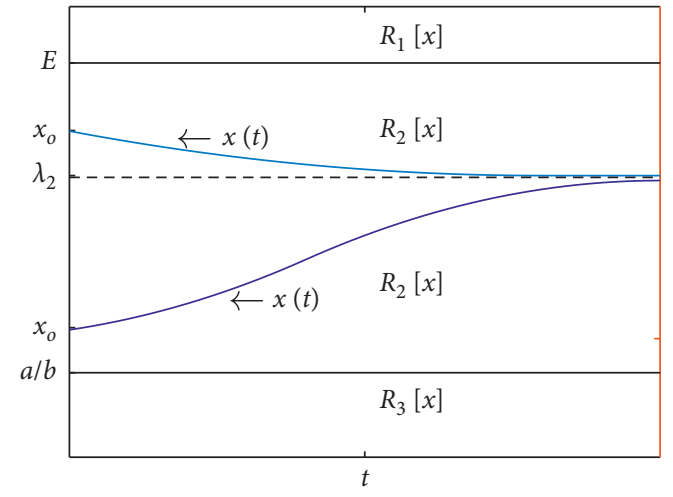

(b)

Figure 2: Continued. 


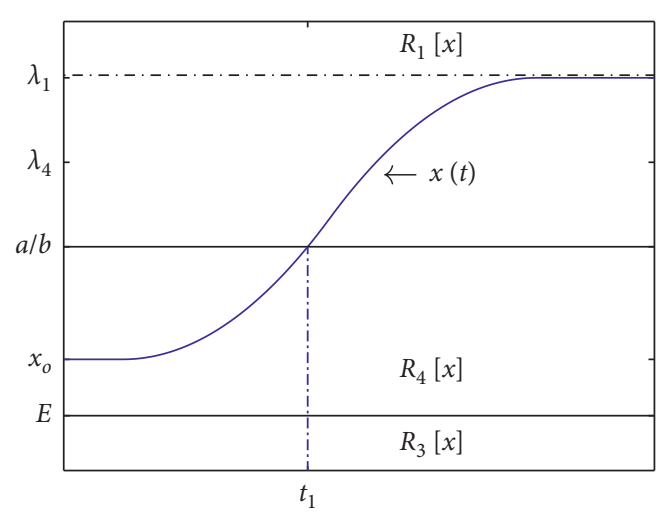

(c)

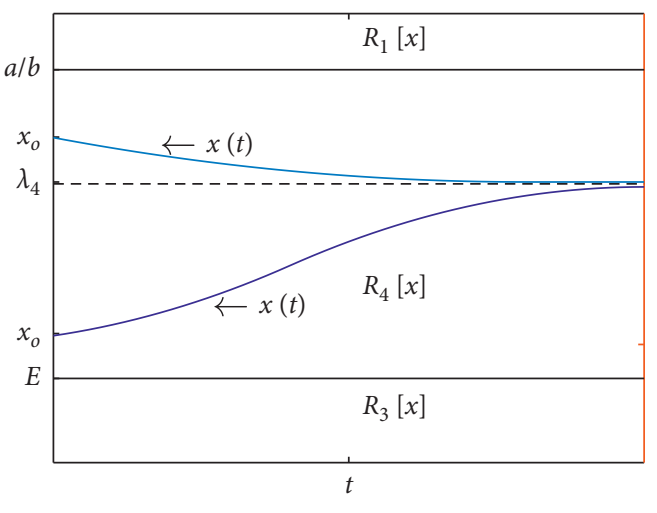

(d)

FiguRE 2: Examples of the construction of the PLF-PM composite trajectory $x(t)$ for $\mathscr{H}[i, 2, k]$ for $i=1,2$, and $k=1,2,3$; referred configuration entitles the $\mathrm{BMS}_{2}:(\beta>\mu a)$ condition. (a) The statement $\left(\left(\lambda_{2}>E\right) \wedge \mathscr{H}[\mathbf{1}, \mathbf{2}, \mathbf{1}]\right) \equiv 1$ holds, $\lambda_{1}$ is a stable equilibrium in the region $\mathfrak{R}_{1}[x]$. (b) Under the $\left(\left(\lambda_{2}<E\right) \wedge \mathscr{H}[1,2,2]\right) \equiv 1$ statement, $\lambda_{2}$ places in region $\Re_{2}[x]$ and becomes a stable equilibrium solution. (c) Whenever $\left(\left(E<\lambda_{4}<a / b\right) \wedge \mathscr{H}[2,2,2]\right) \equiv 1$, the $\lambda_{4}$ equilibrium places in $\mathfrak{R}_{4}[x]$; since $E<x_{0}<a / b, x(t)$ increases, reaching the $a / b$ boundary at time $t_{1}$, and switches to rule $x_{1}(t)$ but since $\lambda_{1}>a / b, x(t)$ increases asymptotically towards $\lambda_{1}$ staying within region $\mathfrak{R}_{1}[x]$. (d) In turn, if $\left(\left(E<\lambda_{4}<a / b\right) \wedge \mathscr{H}[2,2,2]\right) \equiv 1$, then $\lambda_{4}$ stands for a stable equilibrium solution in the region $\mathfrak{R}_{4}[x]$.

equilibrium in the region $\mathfrak{R}_{2}[x]$ (see Figure 2(b)). Under the $\mathscr{H}[\mathbf{2}, \mathbf{2}, \mathbf{2}]$ arrangement, population size $x(t)$ begins in the region $\mathfrak{R}_{4}[x]$ following the $x_{4}(t)$ rule with $\lambda_{4}>E$. If $E<x_{0}<\lambda_{4}<a / b, x(t)$ increases as it asymptotically approaches $\lambda_{4}$. On the other hand, if $E<\lambda_{4}<x_{0}<a / b, x(t)$ decreases while asymptotically approaching $\lambda_{4}$. Therefore, the condition $\left(\left(E<\lambda_{4}<a / b\right) \wedge \mathscr{H}[2,2,2]\right) \equiv 1$ bears a stable equilibrium at $\lambda_{4}$ (see Figure $2(\mathrm{c})$ ). Whenever $\lambda_{4}>a / b$, the $\lambda_{4}$ equilibrium lies within region $\mathfrak{R}_{1}[x]$. Since $E<x_{0}<a / b$, population size increases according to rule $x_{4}(t)$ approaching $\lambda_{4}$ reaching the $a / b$ boundary at time $t_{1}$ switching to rule $x_{1}(t)$, since $\lambda_{1}>a / b$ population size increases asymptotically towards $\lambda_{1}$ staying within region $\mathfrak{R}_{1}[x]$ (see Figure $2(\mathrm{~d})$ ). $\mathscr{H}[\mathbf{1}, \mathbf{2}, \mathbf{3}]$ and $\mathscr{H}[\mathbf{2}, \mathbf{2}, \mathbf{3}]$ bear $x(t)$ increasing from $E<x_{0}$ and could approach asymptotically $\lambda_{4}$ whenever this equilibrium solution lies within region $\mathfrak{R}_{4}[x]$ or $\lambda_{1}$ if $\lambda_{4}>a / b$.

For $\mathscr{H}[\mathbf{1}, \mathbf{3}, \mathbf{1}]$, population size $x(t)$ starts in the region $\mathfrak{R}_{1}[x]$ and controls by $x_{1}(t)$ with equilibrium solution $\lambda_{1}$ satisfying $a / b<\lambda_{1}<E<x_{0}$. Hence, $x(t)$ decreases and attains the $E$ threshold at a time $t_{1}$, transferring to rule $x_{2}(t)$ and then asymptotically approaching the equilibrium solution $\lambda_{2}=a / b$ (see Figure 3(a)). For the arrangement $\mathscr{H}[1,3,2]$, the initial condition sets $x(t)$ to acquire the $x_{2}(t)$ law in $\mathfrak{R}_{2}[x]$ and then it approaches the equilibrium solution $\lambda_{2}$. Correspondingly, the $\mathscr{H}[1,3,3]$ composite $x(t)$ obeys the $x_{3}(t)$ rule in region $\Re_{3}[x]$, but since $\beta=\mu a$, it remains stationary at $x_{0}$. These configurations suggest a maximum limiting effect of resource scarcity. For $\mathscr{H}[\mathbf{2}, \mathbf{3}, \mathbf{1}]$, population size $x(t)$ begins within the region $\Re_{1}[x]$ and since $\lambda_{1}<a / b$ places in region $\Re_{4}[x]$, then $x_{1}(t)$ decreases, intersecting $a / b$ at a time $t_{1}$ where it switches to rule $x_{4}(t)$, ultimately approaching the equilibrium solution $\lambda_{4}=E$. A $\mathscr{H}[2,3,2]$ arrangement sets $E<x_{0}<a / b$, and population size starts within the region $\mathfrak{R}_{4}[x]$ and sets by $x_{4}(t)$ approaching the $\lambda_{4}=E$ equilibrium (see Figure $3(\mathrm{~b})$ ). For
$\mathscr{H}[2,3,3]$, the conditions $x_{0}<E$ and $\beta=\mu a$ set population size beginning within the region $\mathfrak{R}_{3}[x]$, following $x_{3}(t)$ that remains stationary at $x_{0}$. For $\mathscr{H}[\mathbf{3}, \mathbf{3}, \mathbf{1}]$, initial condition sets population size $x(t)$ within the region $\Re_{1}[x]$, following $x_{1}(t)$. Since $\beta=\mu a$ and $E=a / b$ implies $\lambda_{1}=E$, then $x(t)$ decreases to $\lambda_{1}$ (see Figure 3(c)). For $\mathscr{H}[3,3,2]$, we have $x_{0}<E$, and population size starts within the region $\mathfrak{R}_{3}[x]$ and follows to growth law $x_{3}(t)$ but since $\beta=\mu a$, it remains stationary at $x_{0}$ (see Figure 3(d)). Configurations in Figure 3 suggest a maximum limiting effect of resource scarcity on population growth; even a $B M S_{1}$ : $(\beta<\mu a)$ condition does not perform.

In summary, the PLF-PPM's continuous-time global population size trajectory can stand an outstanding array of different performances. It could model population decline whenever the ordering $\beta<\mu a$ holds (see Figure 1). Alternatively, $\beta>\mu a$ could induce conditional stability depending on the achieved parametric arrangement (e.g., Figure 2). Also, the statement $\beta>\mu a$ could entail a purely growing regime for population size $x(t)$ (e.g. $\left(\left(E<\lambda_{4}<a / b\right) \wedge \mathscr{H}[\mathbf{2}, \mathbf{2}, \mathbf{2}] \equiv 1\right)$ shown in Figure $2(\mathrm{~d})$. Furthermore, $\beta=\mu a$ circumstance signposts a maximum effect of resource abatement on population growth (e.g., $\mathscr{H}[3,3,1]$ shown in Figure 3(c)). Moreover, for critical values of $E$ threshold, this configuration could exacerbate Allee effects, and this is due to random influences which could promote vanishing of population size. Furthermore, the ratio $a / b$ in the settings of the PLF-PPM is interpreted as an Allee threshold since $x(t)<a / b$ implies $a x(t)>b x^{2}(t)$, so population size below $a / b$ controls the mortality process as entailed by the $M(x)$ setup (cf. equation (21)). It is also pertinent to emphasize that for model type $M_{1}$, even though population size places below the starvation threshold $E$, its dynamics could take place under an Allee effect regime. Correspondingly, for $M_{2}$ type models, population could evolve in a regime combining both 


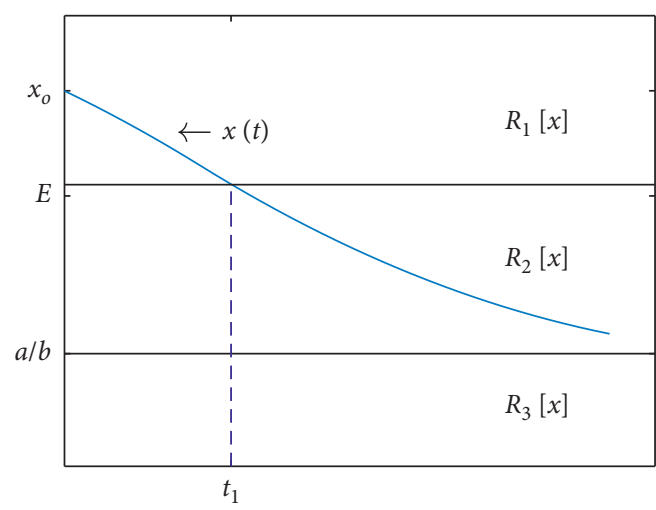

(a)

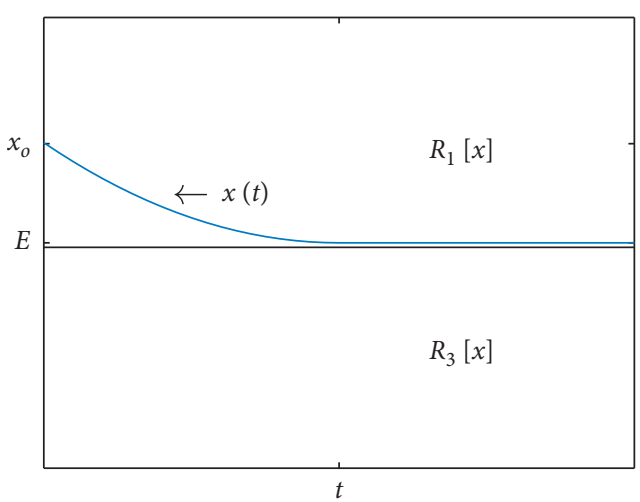

(c)

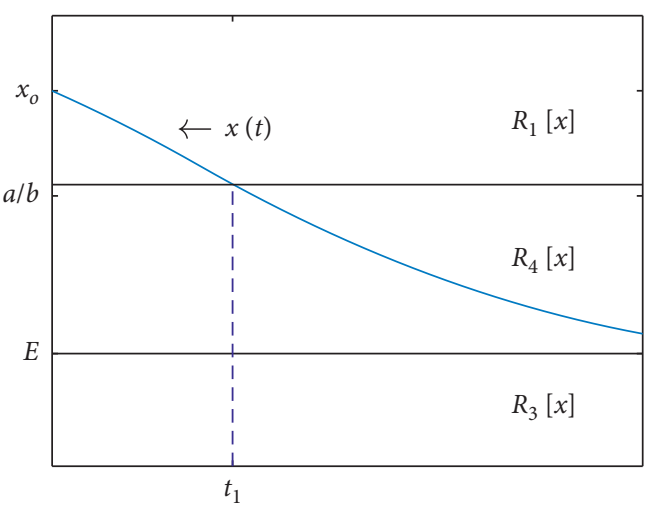

(b)

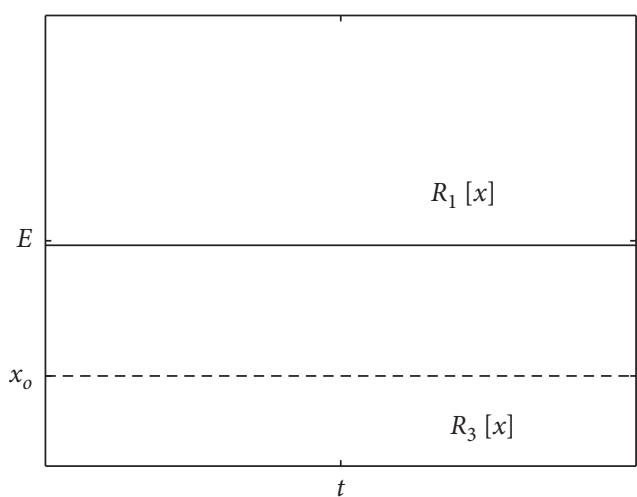

(d)

Figure 3: Examples of the shape of the global trajectory $x(t)$ for $\mathscr{H}[i, 3, k(i)]$ configuration. (a) The $x(t)$ path for the $\mathscr{H}[\mathbf{1}, \mathbf{3}, \mathbf{1}]$ conjunction population size $x(t)$ starts in the region $\mathfrak{R}_{1}[x]$ and approaches asymptotically the equilibrium solution $\lambda_{2}=a / b$. (b) For $\mathscr{H}[2,3,2]$, population size $x(t)$ begins in the region $\Re_{1}[x]$ according to $x_{1}(t)$ and decreases and then switches to rule $x_{4}(t)$ to approach asymptotically $\lambda_{4}=E$. (c) $\mathscr{H}[3,3, \mathbf{1}]$ ordering sets $x(t)$ initially within the region $\mathfrak{R}_{1}[x]$ and then $x(t)$ decreases to $\lambda_{1}=E$. (d) For $\mathscr{H}[3,3,2]$, we have $x_{0}<E$, and population size starts within the region $\mathfrak{R}_{3}[x]$ and follows growth law $x_{3}(t)$ but since $\beta=\mu a$, it remains stationary at $x_{0}$.

starvation and Allee effects. Consequently, by adapting the number of factors defining $N(x)$ and $M(x)$ in equation (17) so as to suit specific modeling aims, we could endure the PLF-PPM suitable predictive strength. In what follows, we explain the performance of the PLF-PPM model of equation (22) as an exploratory tool given different datasets.

\section{Study Cases}

In what follows, we explain the performance of the PLFPPM model of equation (22) as an exploratory tool given different datasets. We address data of Armstrong [7] on the growth of populations of asexual Dugesia tigrina, data reported by Huisman [8] on developing experimental populations of the unicellular green algae Chlorella vulgaris, a study by Davidson [9] on a sheep population introduced in Tasmania, data by Pearl [10] on the growth of Drosophila melanogaster, and data reported by Hughes and Tanner [11] on the slow decline of an Agaricia agaricites population on Jamaican reefs. Fitted parameters, associated standard deviations, and concordance correlation coefficient values [12] are given in Table 3. All required PLF-PPM fits were achieved by using the Berkeley Madonna Software Version
8.3.18. Besides, acquiring the resource abatement function $R(x)$ relied on using equation (18) setting $E=F_{0} / 2 k$ for $k=1$, which assures getting a proxy for the maximum depletion rate.

Armstrong [7] maintained populations of asexual Dugesia tigrina, in an arrangement of finger bowls. Bowls contained $120 \mathrm{ccs}$ of water each and were kept at a temperature of $25^{\circ} \mathrm{C}$. Every other day, each population received 0.1 ccs of freshly killed brine shrimp. The bowls were cleaned at the end of the feeding period. The initial population size in every bowl amounted to 35 worms. Reproduction occurred only by transverse fission, with each worm dividing to produce a tail that developed into a new individual. For data assembly, a tail was any recent fission product not adequately developed to consume food. In the experiment, tails were added to the population's artificially increasing reproductive efficiency. Because this method amounts to exogenous addition of biomass, we focused on data of population control named 1-0 without tails added to fit the PLF-PM. The plot of the CCC $=0.9920$ global trajectory is shown in Figure 4(a). Associated estimated parameter values are presented in Table 3. Acquired orderings were $\beta>\mu a$ and $x_{o}<a / b<\lambda_{1}<\lambda_{2}<E<\lambda_{4}$ corresponding to a $\mathscr{H}[\mathbf{1}, \mathbf{2}, \mathbf{3}]$ arrangement composing regions 


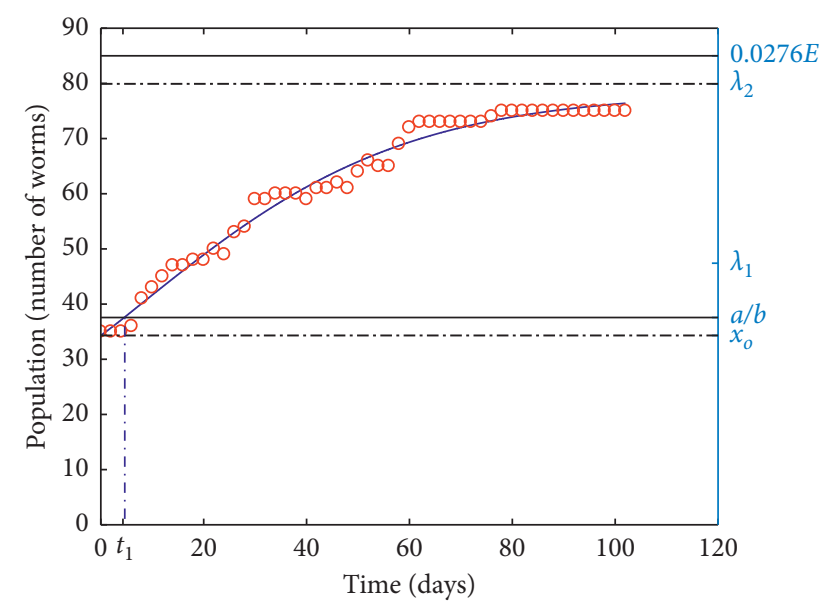

(a)

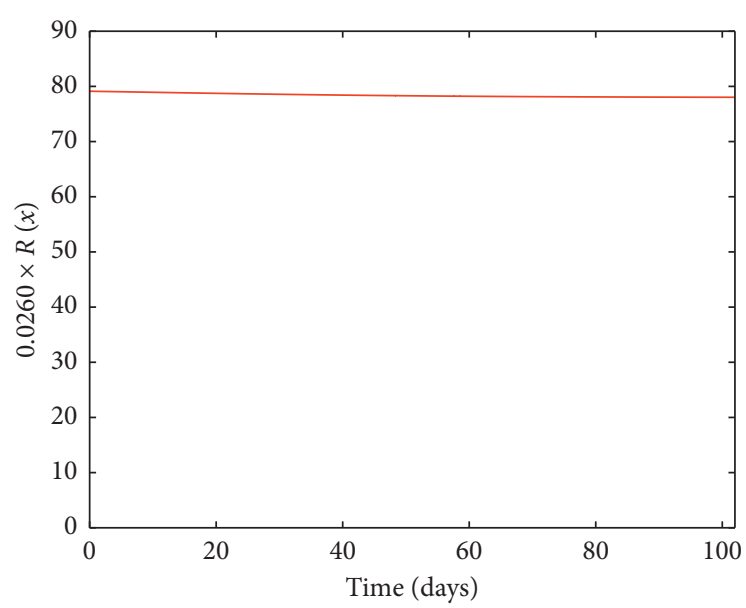

(b)

Figure 4: Fit of the PLF-PPM to data of Armstrong [7] relates to the growth of an experimental population of planarian Dugesia tigrina. (a) Fitted trajectory lines. (b) Proportion $(0.0260 \times R(x))$ of resource abatement function.

$\mathfrak{R}_{1}[x], \mathfrak{R}_{2}[x]$, and $\mathfrak{R}_{3}[x]$. Population size places initially within the region $\Re_{3}[x]$ and acquires the form $x_{3}(t)$ until it reaches the upper boundary at $a / b$ at time $t_{1}=4.43$ where it gets into the region $\mathfrak{R}_{2}[x]$ switching to rule $x_{2}(t)$, approaching the asymptotic limit $\lambda_{2}$. Since dynamics mainly describe the region $\mathfrak{R}_{2}[x]$, mortality could perhaps be explained by cannibalism induced by high intraspecific competition. The author inferred about food scarcity for population levels near the equilibrium. Nevertheless, the PLF-PM identifies an energy threshold $E$ well above the equilibrium level, which explains the shape of the resource depletion trajectory proxy shown in Figure 4(b). Therefore, population regulation is solely controlled by density-dependent mortality.

Huisman [8] studied the growth of experimental populations of the unicellular green algae Chlorella vulgaris. Populations were raised in continuous cultures incubated at $20^{\circ} \mathrm{C}$ and administered with plentiful nutrients and $\mathrm{O}_{2}$. Thus, growth limitation is exclusively associated with incident light energy. We handpicked data on population $G$. Figure 5(a) shows the global trajectory associated with the CCC $=0.9946$ fit of the PLF-PM. The values of estimated parameters are presented in Table 3. Acquired parametric orderings were $\beta>\mu a$ and $x_{o}<a / b<\lambda_{2}<E$ conforming to $\mathscr{H}[\mathbf{1}, \mathbf{2}, \mathbf{3}]$ composing regions $\mathfrak{R}_{1}[x], \mathfrak{R}_{2}[x]$, and $\mathfrak{R}_{3}[x]$. Population size places initially within the region $\Re_{3}[x]$ and rules by $x_{3}(t)$ until it reaches the upper boundary at $a / b$ at time $t_{1}=3.7$ where it goes into the region $\Re_{2}[x]$ switching to rule $x_{2}(t)$, approaching the asymptotic limit $\lambda_{2}$. The equilibrium value $\lambda_{2}$ was practically attained at the end of the $18^{\text {th }}$ day, with regulation by recruitment of new individuals and mortality dominated by individual properties. This photosynthetic organism synthesizes biomass based on a combination of factors like light, $\mathrm{CO}_{2}$, and nutrients. The threshold $\mathbf{E}$ could be conceived as the biomass size such that the aggregated life enduring factors become limiting factors. Because there were sufficient $\mathrm{CO}_{2}$ and nutrients in this case, the $E$ threshold could be interpreted as the level of available light energy. Our analysis sustains the assumption in [8] that population growth in Chlorella vulgaris is limited exclusively by incident light. Therefore, the value of the $E$ threshold estimated by the fit of the PLF-PPM determines a resource abatement function that suggests that the amount of incident light for the G-labeled population in Huisman's experiment was not limiting (see Figure 5(b)); this is because the equivalent of $E$ in biomass units lies well above the equilibrium level $\lambda_{2}$.

Davidson [9] studied a sheep population introduced in Tasmania. We acquired related proxy data from [13] representing the averages of the number of individuals taken on periods of 5 years. The recorded data span a total of 120 years. The first average corresponds to 1814-1819, and we arranged for 1819 to stand for year zero. The global trajectory acquired from the CCC $=0.9484$ fit of the PLF$\mathrm{PM}$ is shown in Figure 6(a). The obtained parametric orderings were $\beta>\mu a$ and $x_{o}<a / b<\lambda_{1}<\lambda_{2}<E<\lambda_{4}$, associated to a $\mathscr{H}[\mathbf{1}, \mathbf{2}, \mathbf{3}]$ arrangement that composes regions $\mathfrak{R}_{1}[x], \mathfrak{R}_{2}[x]$, and $\mathfrak{R}_{3}[x]$. Population size places initially within the region $\mathfrak{R}_{3}[x]$ and progressed according to rule $x_{3}(t)$. The fit points out that within the region $\mathfrak{R}_{3}[x]$, population growth was slow at the beginning, which suggests that reproduction was limited by a reduced number of individuals. Population size crossed the $a / b$ threshold at the beginning of year 6 (1825). Population growth switched to rule $x_{2}(t)$, associated with recruitment and mortality controlled by population size. Thus, fitted trajectory increased approaching the equilibrium $\lambda_{2}$ placing inside $\mathfrak{R}_{2}[x]$ (see Figure 6(a)). Besides, it can be learned from Figure 6(b) that in spite of surpassing two records and another two almost reaching the $E$ threshold, their influence was not strong enough so as to change the damped oscillating trend about $\lambda_{2}$ imposed by rule $x_{2}(t)$. Indeed, the dominance of the dynamic arrangement endured by the $x_{2}(t)$ rule stayed over some 


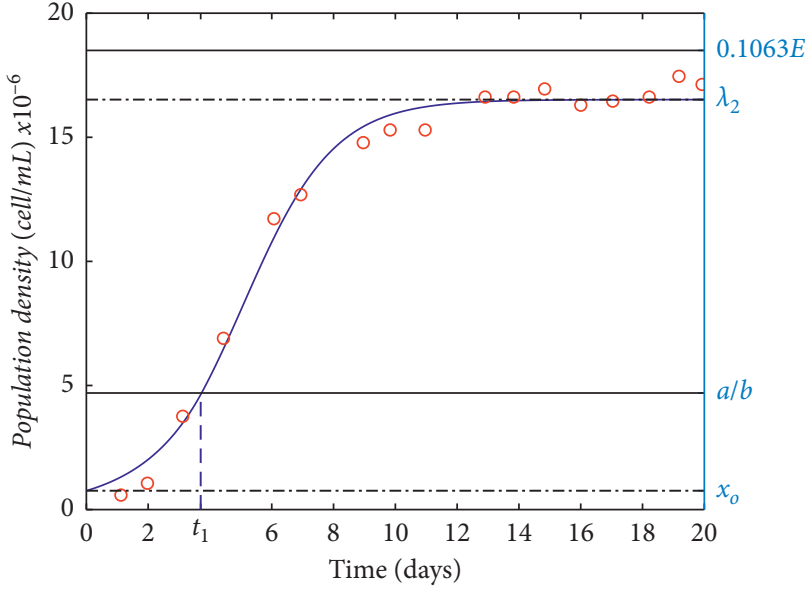

(a)

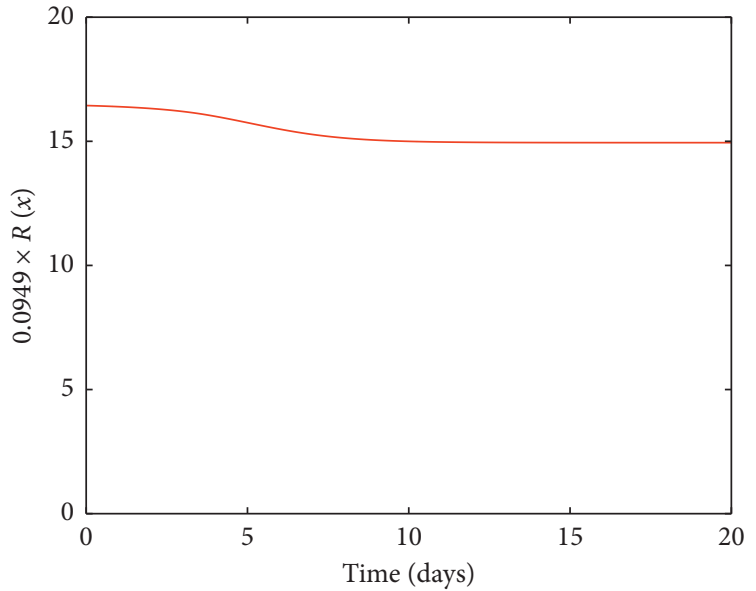

(b)

Figure 5: Fit of the PLF-PPM to data taken from Huisman [8] relates to the growth of an experimental population of the unicellular green algae, Chlorella vulgaris. (a) Fitted trajectory lines. (b) Proportion $(0.0949 \times R(x))$ of resource abatement function.

TABLE 3: Estimated values of initial population size $x_{9}$ and basic parameter $a, b, \beta, \mu, E$, produced by fitting the PLF-PPM of equation (22) to the listed datasets.

\begin{tabular}{|c|c|c|c|c|c|c|c|c|c|c|c|}
\hline \multirow{2}{*}{ Dataset } & \multicolumn{11}{|c|}{ Fitted parameters } \\
\hline & $x_{o}$ & $A$ & $b$ & $B$ & $\mu$ & $E$ & $a / b$ & $\lambda_{1}$ & $\lambda_{2}$ & $\lambda_{4}$ & CCC \\
\hline \multirow{2}{*}{ Armstrong [7] } & 34.31 & 3.59 & 0.09 & 0.03 & 0.0050 & 3077.43 & 39.88 & 47.4100 & 79.91 & $6.5 e+03$ & 0.9920 \\
\hline & 0.06 & 0.38 & 0.06 & 0.00 & 0.0003 & 625.18 & - & - & - & - & - \\
\hline \multirow{2}{*}{ Huisman [8] } & 0.75 & 186.53 & 39.71 & 0.68 & 0.0010 & 174 & 4.69 & $2.12 e+3$ & 16.51 & $2.1 e+3$ & 0.9946 \\
\hline & 0.11 & 49.65 & 15.31 & 0.02 & 0.0002 & 84.56 & - & - & - & - & - \\
\hline \multirow{2}{*}{ Pearl [10] } & 3.96 & 1.89 & 0.08 & 0.25 & 0.0092 & 628.30 & 22.18 & 38.27 & 318.28 & $9.0 e-3$ & 0.9913 \\
\hline & 1.15 & 0.48 & 0.00 & 0.01 & 0.0001 & 37.48 & - & - & - & - & - \\
\hline \multirow{2}{*}{ Davidson [9] } & 85.73 & 146 & 0.56 & 0.18 & $1.94 e+4$ & 1974 & 260.71 & $1.02 e+3$ & $1.69 e+3$ & $1.2 e+4$ & 0.9484 \\
\hline & 58.10 & 41.79 & 1.75 & 0.02 & 0.0000 & 178.63 & - & - & - & - & - \\
\hline \multirow{2}{*}{ Hughes and Tanner [11] } & 213.42 & 1186.56 & 1.57 & 0.09 & $1.64 e+4$ & 376.55 & 755.77 & 180.74 & 360.98 & 752.05 & 0.9340 \\
\hline & 1.5153 & 309.59 & 1.95 & 0.05 & $9.49 e-5$ & 104.61 & - & - & - & - & - \\
\hline
\end{tabular}

Records right below parameter estimates correspond to associated standard errors. Calculated $a / b$ ratio, equilibria $\lambda_{1}, \lambda_{2}, \lambda_{4}$, and concordance correlation coefficient (CCC) values are also displayed.

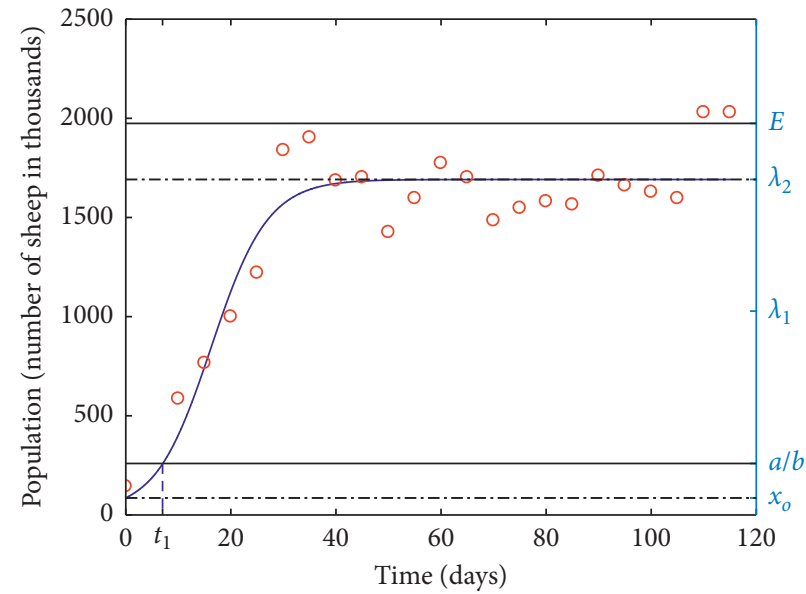

(a)

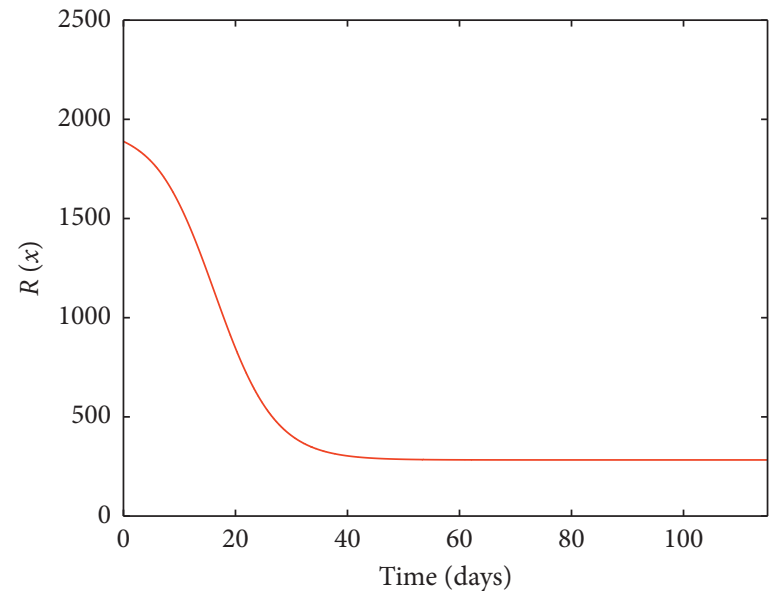

(b)

Figure 6: Fit of the PLF-PPM to data of Davidson [9] relates to the growth of a sheep population introduced in Tasmania. (a) Fitted trajectory lines. (b) Resource abatement function $R(x)$. 


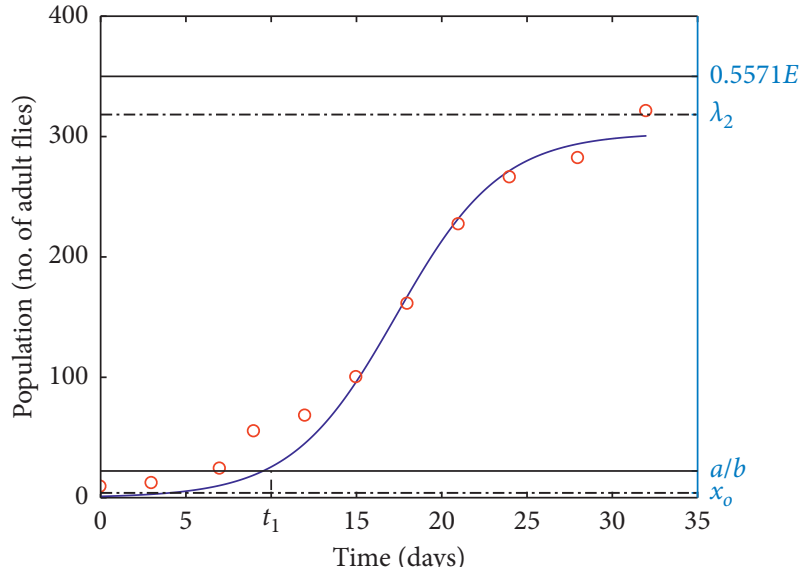

(a)

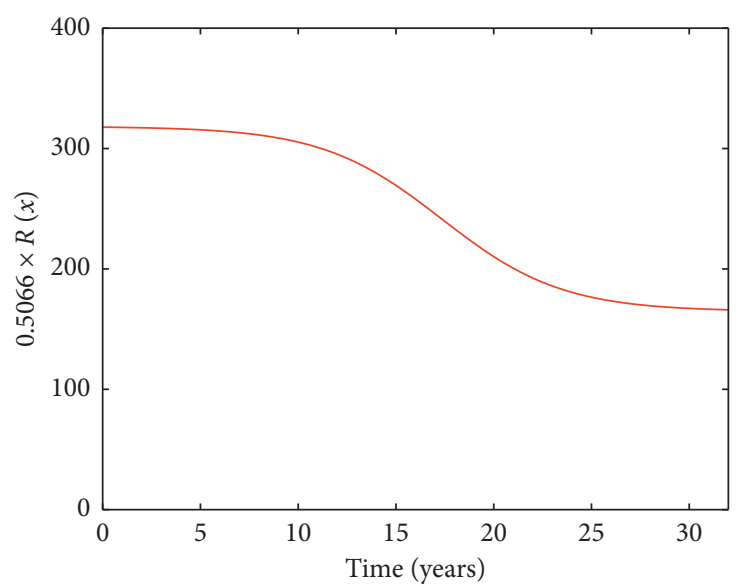

(b)

FIgURE 7: Fit of the PLF-PPM to data of Pearl [10] relates to the growth of an experimental population of the fruit fly Drosophila melanogaster. (a) Fitted trajectory lines. (b) Proportion $(0.5066 \times R(x))$ of resource abatement function $R(x)$.

time long enough to induce the trend to equilibrium suggested by the PLF-PPM fit.

We now consider the construction of the global trajectory $x(t)$ associated with a fit of the PLF-PM to data of [10], taken from [14]. Pearl [10] maintained populations of Drosophila melanogaster in bottles using yeast as food and fitted a logistic curve to the associated data expressed as the counted number of adult flies in a particular day. Sang [15] criticized the procedure, quarreling that the experiments did not keep the yeast constant but as a growing population on its own. The plots associated with the PLF-PPM fit displayed in Figure 7 (a) produced the parameter orderings $\beta>\mu a$ and $x_{0}<a / b<E<\lambda_{2}$ that conform to a $\mathscr{H}[1,2,3]$ arrangement, that is, a model type $M_{1}$ with $\mathrm{BMS}_{2}:(\beta>\mu a)$ and $\mathrm{IC}_{13}:\left(x_{o}<a / b\right)$. Phase arrangement includes regions $\mathfrak{R}_{1}[x], \mathfrak{R}_{2}[x]$, and $\mathfrak{R}_{3}[x]$. At the beginning of the growth process, $x(t)$ stays in the region $\Re_{3}[x]$ but the statement $\mathrm{BMS}_{2}:(\beta>\mu a) \equiv 1$, forces associating a rule $x_{3}(t)$ to increase, and then it reaches the $a / b$ boundary at $t_{1}=10$, thereby entering the region $\mathfrak{R}_{2}[x]$ and then progressing asymptotically to $\lambda_{2}$ following rule $x_{2}(t)$. The fit shows that the fly population is entirely controlled by density-dependent mortality. In contrast to Sang [15], the PLF-PPM fit demonstrates that energy did not play a decisive role $\left(\left(E>\lambda_{2}\right)\right.$; also, see Figure $\left.7(b)\right)$, so we can close that variations in feeding energy did not determine the dynamics since otherwise $x_{1}(t)$ would compose the global trajectory $x(t)$. The application of the FDM sustains the assumption that in Pearl's experiment, energy does not provide a criterion to define a carrying capacity. Furthermore, the equilibrium $\lambda_{2}$ is not expressed in terms of E but solely as a function of the scaling parameters $\beta, \mu$, and $b$.
Hughes and Tanner [11] reported a slow decline of the coral population on Jamaican reefs over 16 years. We fitted the PLF-PM to data on Agaricia agaricites colonies recorded during 1977-1993. The authors established that local extinction is explained by increased mortality rates plus the adverse effects of two hurricanes, Allen (in 1980) and Gilberto (in 1988). Also relevant in explaining the decline was the impossibility of recovery because of a recruitment failure. Figure 8(a) shows the global trajectory associated with the fit CCC $=0.9340$ of the PLF-PM. The values of the parameter estimates are given in Table 3 . Acquired parametric orderings were $\beta<\mu a$ and $x_{o}<\lambda_{2}<E<\lambda_{1}<a / b$ conforming to a $\mathscr{H}[2,1,3]$ arrangement and composing regions $\mathfrak{R}_{1}[x], \mathfrak{R}_{2}[x]$, and $\mathfrak{R}_{3}[x]$. Population size places initially within the region $\mathfrak{R}_{3}[x]$ and rules by $x_{3}(t)$. Food was not limiting. Perhaps perturbation set its level under the upper boundary of the $\mathfrak{R}_{3}[x]$ region where Allee effects dominate mortality. Then, possibly a recruitment failure impeded compensation to the abatement effects linked to the $\beta<\mu a$ ordering. Then, our analysis corroborates the assumption of Hughes and Tanner [11]. In any event, recruitment failure could not associate with resource reduction (see Figure 8(b)).

The PLF-PPM displayed high reproducibility strength in all performed fits, as it derives from the high CCC values included in Table 3. Moreover, parallel plots of resource abatement explain that fitted values of initial resource availability thresholds E explain the occurrence of recorded equilibrium levels. Results point towards the consistency of the PLF-PPM as an exploratory tool. Nevertheless, the direct fitting procedure endured by the Berkeley Madonna software relied upon apportioning initial values of 


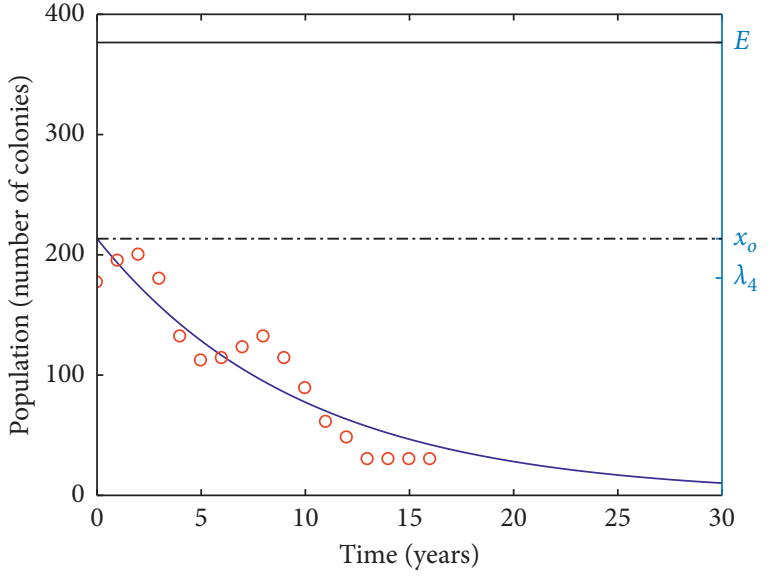

(a)

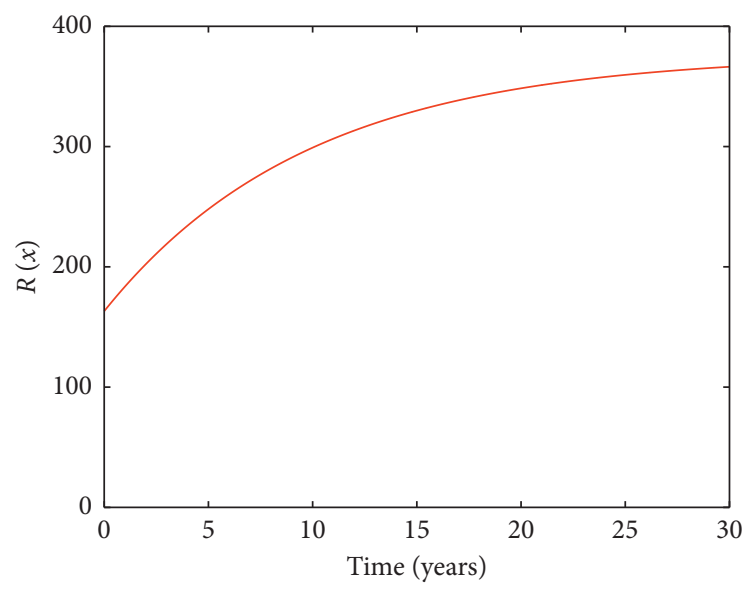

(b)

Figure 8: Fit of the PLF-PM to data of Hughes and Tanner [11] relates to the decline of Agaricia agaricites coral population on Jamaican reefs. (a) Fitted trajectory lines. (b) Resource abatement function $R(x)$.

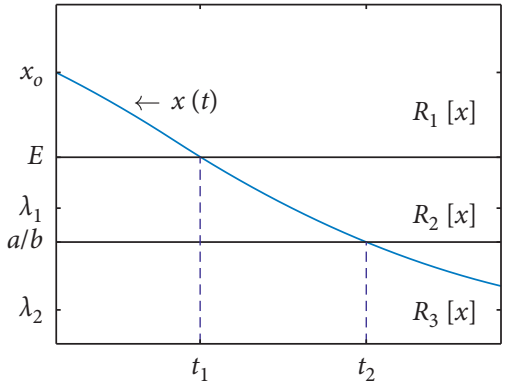

(a)

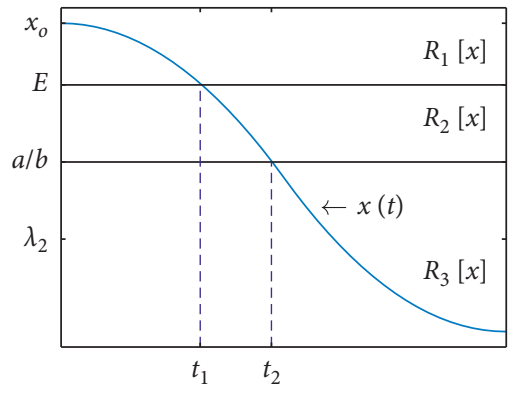

(b)

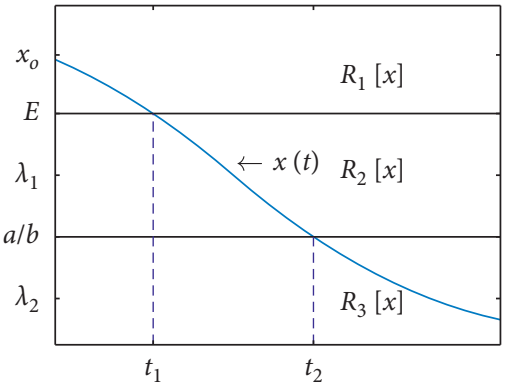

(c)

Figure 9: The PLF-PPM composite trajectory $x(t)$ for the $\mathscr{H}[\mathbf{1}, \mathbf{1}, \mathbf{1}]$ configuration. (a) $a / b<\sqrt{\beta E / \mu b}<E$ derived from the auxiliary ordering $E>\mu a^{2} / \beta b$. This panel shows that the global trajectory $x(t)$ reaches the $E$ threshold at a time $t_{1}$. (b) Population size keeps decreasing, so it touches the $a / b$ frontier. (c) Since $\beta<\mu a$, the population becomes extinct.

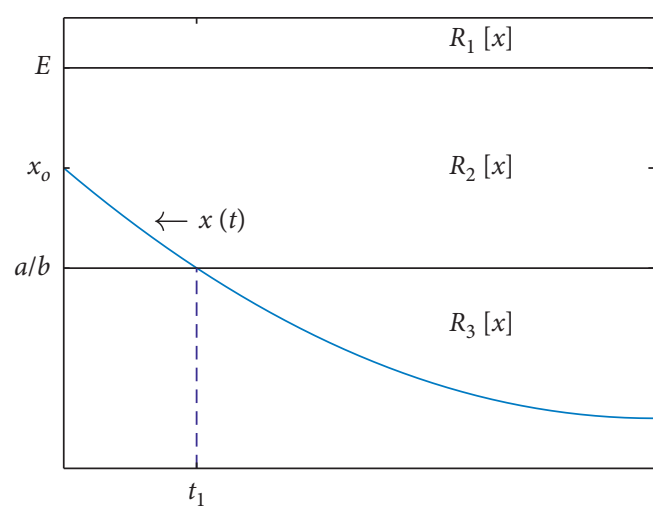

(a)

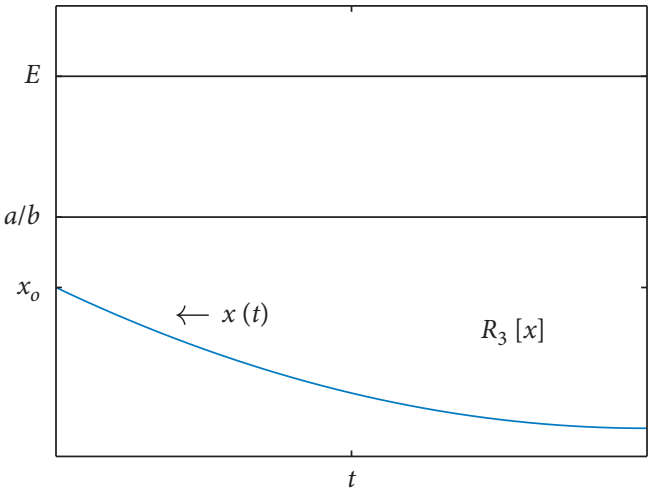

(b)

Figure 10: The PLF composite trajectory $x(t)$ for the $\mathscr{H}[\mathbf{1}, \mathbf{1}, \mathbf{2}]$ configuration. (a) The global trajectory $x(t)$ decreases from $x_{o}$ until it reaches the $a / b$ threshold at time $t_{1}$. Since $\beta<\mu a$, population size keeps decreasing until it becomes extinct. (b) Extinction as determined by the $\mathscr{H}[1,1,3]$ arrangement. 
parameter estimates. Necessarily, this brought about high sensibility associated to local minimum problems at the non-linear acquisition of final estimates. Detected inconveniences suggest revision aimed at adapting techniques that could lessen the experienced parameter estimation burden.

4.1. Outline of the Discrete-Time Setup of the PLF-PPM. Although this paper focuses on addressing the analysis of the PLF-PPM arrangement trajectories on continuous time, we consider it pertinent at this setting to outline the associated discrete-time form. For this reason, we let $f_{i}\left(x_{n}\right)$ denote the discrete-time form of the phase model $f_{i}(x)$ as given by equation (22) for $i=1,2,3,4$. Also, let $\mathfrak{R}_{i}\left[x_{n}\right]$ be the corresponding range of application of the rule $f_{i}\left(x_{n}\right)$. Then, if $\chi\left(\mathfrak{R}_{1}\left[x_{n}\right]\right)$ stands for the characteristic function of $\mathfrak{R}_{1}\left[x_{n}\right]$, we get

$$
x_{n+1}=x_{n}+\sum_{i=1}^{4} \chi\left(\Re_{i}\left[x_{n}\right]\right) f_{i}\left(x_{n}\right),
$$

for $n=0,1,2, \ldots$, with $x_{0}$ being placed on one of the regions $\mathfrak{R}_{i}\left[x_{n}\right]$. Then,

$$
\left.\begin{array}{ll}
f_{1}\left(x_{n}\right)=\beta E-\mu b x_{n}^{2} & \mathfrak{R}_{1}\left[x_{n}\right]=\left\{x_{n} \mid\left(x_{n}>E\right) \wedge\left(x_{n}>\frac{a}{b}\right)\right\} \\
f_{2}\left(x_{n}\right)=\beta x_{n}-\mu b x_{n}^{2} & \mathfrak{R}_{2}\left[x_{n}\right]=\left\{x_{n} \mid\left(x_{n}<E\right) \wedge\left(x_{n}>\frac{a}{b}\right)\right\}
\end{array}\right\} .
$$

change of variable $(r / s) z_{n}=x_{n}$ will bring the logistic map as a submodel composing the seek for discrete-time formulation. Concomitantly, equation (25) transforms into

where

$$
\begin{array}{ll}
\theta_{1}\left(z_{n}\right)=\frac{s(1-r) E}{r}+z_{n}\left(1-r z_{n}\right), & \mathfrak{R}_{1}\left[z_{n}\right]=\left\{z_{n} \mid\left(z_{n}>\frac{s E}{r}\right) \wedge\left(z_{n}>\frac{s a}{r b}\right)\right\} \\
\theta_{2}\left(z_{n}\right)=r z_{n}\left(1-z_{n}\right) & \mathfrak{R}_{2}\left[z_{n}\right]=\left\{z_{n} \mid\left(z_{n}<\frac{s E}{r}\right) \wedge\left(z_{n}>\frac{s a}{r b}\right)\right\} \\
\theta_{3}\left(z_{n}\right)=\left(r-\frac{s a}{b}\right) z_{n} & \mathfrak{R}_{3}\left[z_{n}\right]=\left\{z_{n} \mid\left(z_{n}<\frac{s E}{r}\right) \wedge\left(z_{n}<\frac{s a}{r b}\right)\right\} \\
\theta_{4}\left(z_{n}\right)=\frac{s(r-1) E}{r}+\left(1-\frac{s a}{b}\right) z_{n} & \mathfrak{R}_{4}\left[z_{n}\right]=\left\{z_{n} \mid\left(z_{n}>\frac{s E}{r}\right) \wedge\left(z_{n}<\frac{s a}{r b}\right)\right\}
\end{array} .
$$

Equivalently, 


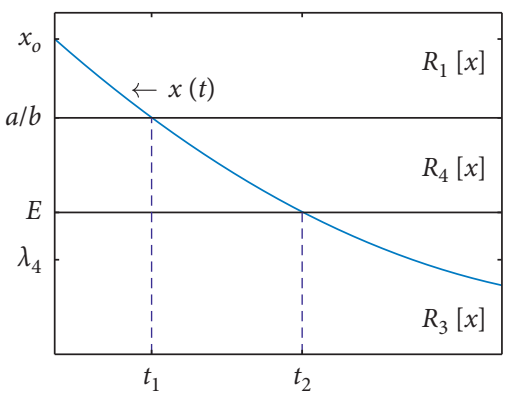

(a)

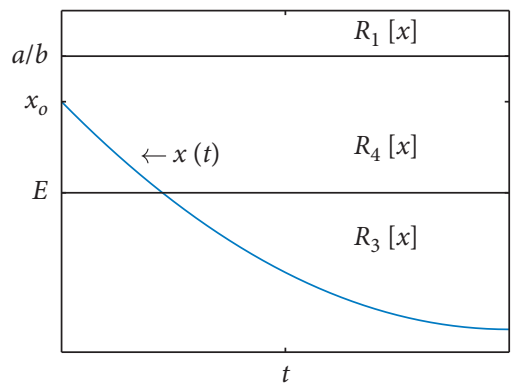

(b)

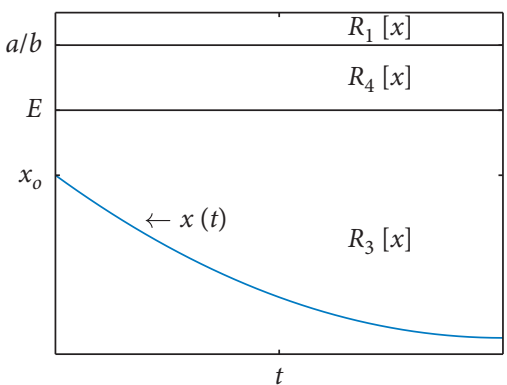

(c)

FiguRE 11: The shape of the PLF composite trajectory $x(t)$ under an extinction configuration. (a) The global trajectory $x(t)$ for the $\mathscr{H}[\mathbf{2}, \mathbf{1}, \mathbf{1}]$ decreases from $x_{o}>a / b>E$ until it reaches the $a / b$ threshold at time $t_{1}$. The $x(t)$ trajectory keeps decreasing, and it hits the $E$ threshold at a particular time $t_{2}$. Later, since $\beta<\mu a$, population size keeps decreasing until it becomes extinct. (b) Case $\mathscr{H}[2, \mathbf{1}, 2]$ where population size decreases from $E<x_{o}<a / b$ and reaches the $E$ boundary at a time $t_{1}$ and then it progresses to extinction. (c) $\mathscr{H}[2, \mathbf{1}, 3]$ configuration, where departing from $x_{o}<E<a / b$, the population becomes extinct.

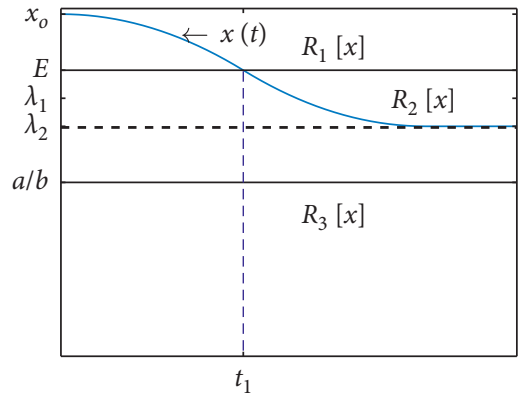

(a)

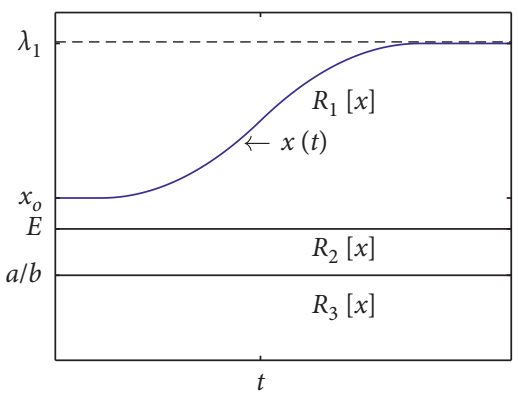

(b)

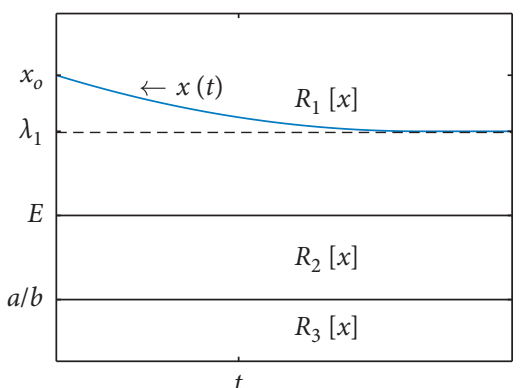

(c)

FIGURE 12: The shape of the PLF composite trajectory $x(t)$ for the $\mathscr{H}[\mathbf{1}, \mathbf{2}, \mathbf{1}]$ configuration. (a) The condition $\left(\left(\lambda_{2}<E\right) \wedge \mathscr{H}[\mathbf{1}, \mathbf{2}, \mathbf{1}]\right) \equiv 1$, where the trajectory departs from $x_{0}>E>a / b$ and decreases as it approaches asymptotically the equilibrium solution $\lambda_{2}$ placed in region $\mathfrak{R}_{2}[x]$. (b) In the case $\left(\left(\lambda_{2}>E\right) \wedge \mathscr{H}[1,2,1]\right) \equiv 1$, departing from $E<x_{o}<\lambda_{1}$, the trajectory increases and asymptotically approaches $\lambda_{1}$. (c) $E<\lambda_{1}<x_{o}$, where population size decreases to $\lambda_{1}$, which establishes $\lambda_{1}$ as a stable equilibrium in the region $\mathfrak{R}_{1}[x]$.

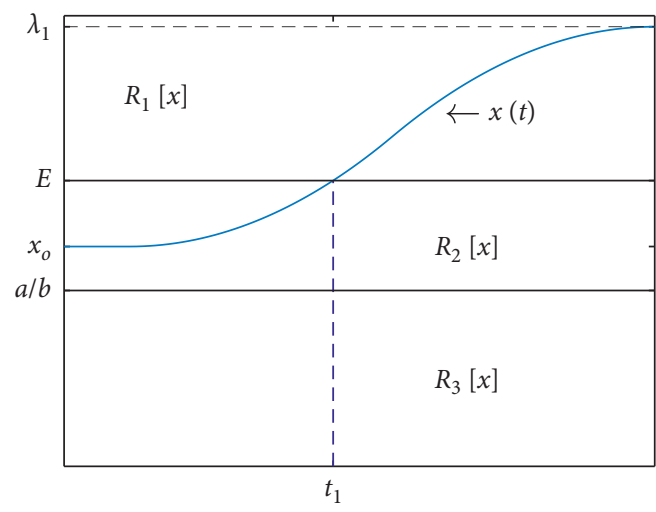

(a)

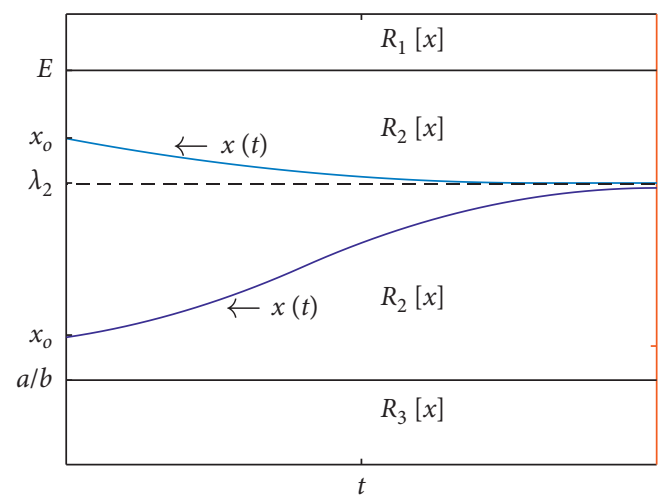

(b)

Figure 13: The shape of the PLF composite trajectory $x(t)$ for the $\mathscr{H}[\mathbf{1}, \mathbf{2}, \mathbf{2}]$ configuration. (a) The case $\left(\left(\lambda_{2}>E\right) \wedge \mathscr{H}[\mathbf{1}, \mathbf{2}, 2]\right) \equiv 1$ where the trajectory departs from $a / b<x_{o}<E<\lambda_{2}$ and increases through the $\Re_{2}[x]$ region as it approaches asymptotically the equilibrium solution $\lambda_{2}$ placed in region $\Re_{1}[x]$, so it will switch at a time $t_{1}$ to rule $x_{1}(t)$ approaching $\lambda_{1}$. (b) The case $\left(\left(\lambda_{2}<E\right) \wedge \mathscr{H}[1,2,2]\right) \equiv 1$. The population size approaches the stable equilibrium $\lambda_{2}$ placed inside $\mathfrak{R}_{2}[x]$. 


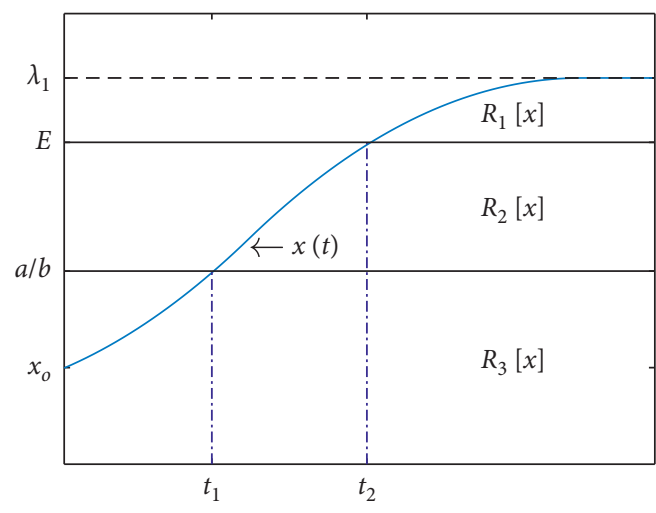

(a)

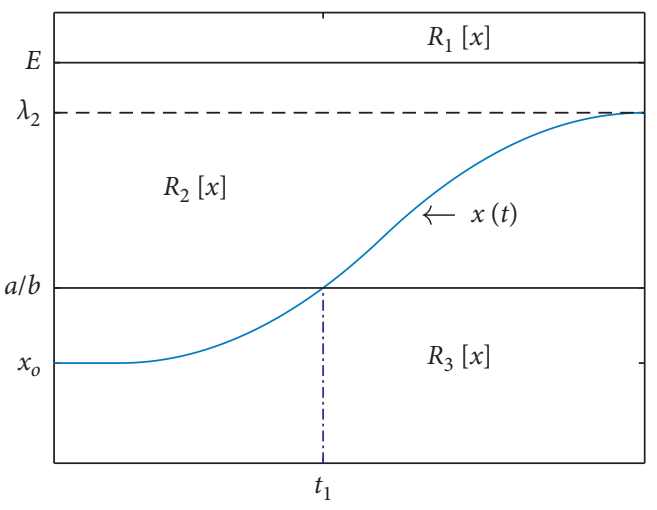

(b)

FIGURE 14: The comportment of the PLF composite trajectory $x(t)$ for the $\mathscr{H}[\mathbf{1}, \mathbf{2}, 2]$ configuration. (a) The case $\left(\left(\lambda_{2}>E\right) \wedge \mathscr{H}[\mathbf{1}, 2,2]\right) \equiv 1$; the relationship $x_{o}<a / b<E<\lambda_{2}$ holds, so the population size $x(t)$ places initially within the region $\Re_{3}[x]$ and grows according to the $x_{3}(t)$ trajectory; then, at a time $t_{1}, x(t)$ reaches $E$, so dynamics are now controlled by $x_{1}(t)$ and consequently will asymptotically approach the $\lambda_{1}$ threshold placed in $\Re_{1}[x]$. (b) $\left(\left(\lambda_{2}<E\right) \wedge \mathscr{H}[1,2,3]\right) \equiv 1$; the inequality $x_{0}<a / b<\lambda_{2}<E$ holds, and population size is ruled by $x_{3}(t)$ until it reaches $a / b$ and switches to $x_{2}(t)$, and then it remains in $\mathfrak{R}_{2}[x]$ approaching the asymptotic limit $\lambda_{2}$.

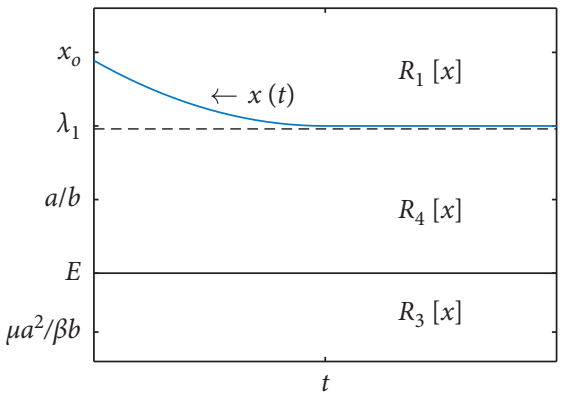

(a)

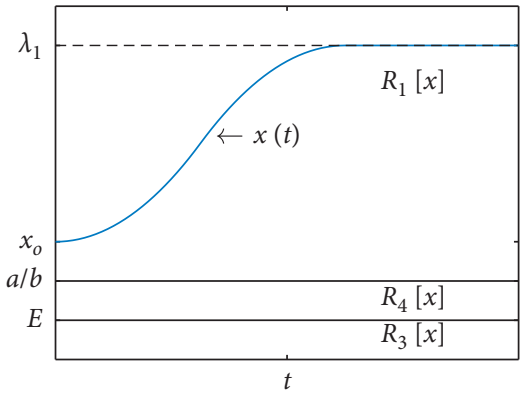

(b)

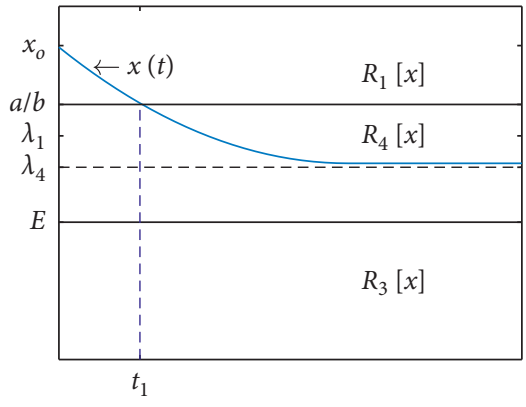

(c)

FIGURE 15: The construction of the global trajectory $x(t)$ under the $\mathscr{H}[2,2,1]$ arrangement. (a) Since $x_{o}>a / b$, population size initiates dynamics within the region $\Re_{1}[x]$ according to the $x_{1}(t)$ rule that bears the equilibrium solution $\lambda_{1}>E$. If $E<a / b<\lambda_{1}<x_{0}$, population size decreases as it approaches $\lambda_{1}$. (b) If $E<a / b<x_{0}<\lambda_{1}$, population size increases approaching $\lambda_{1}$ asymptotically. (c) Whenever $E<\lambda_{1}<a / b<x_{0}$, population size initiates within the region $\Re_{1}[x]$ and decreases towards $\lambda_{1}$, entering into the region $\Re_{4}[x]$ at a time $t_{1}$ and then switching to rule $x_{4}(t)$, but $\beta / \mu a>1$, and then $E<\lambda_{4}<a / b<x_{o}$, so $x(t)$ keeps decreasing as it approaches asymptotically $\lambda_{4}$.

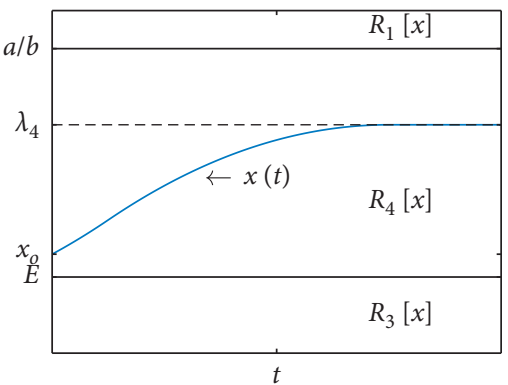

(a)

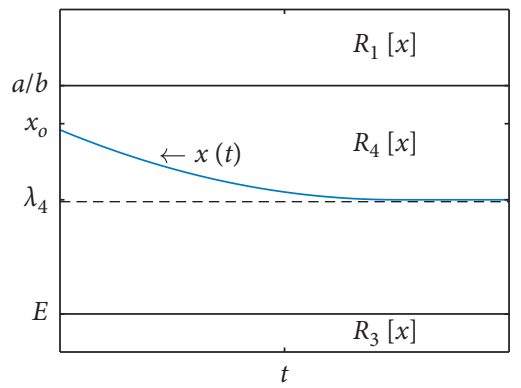

(b)

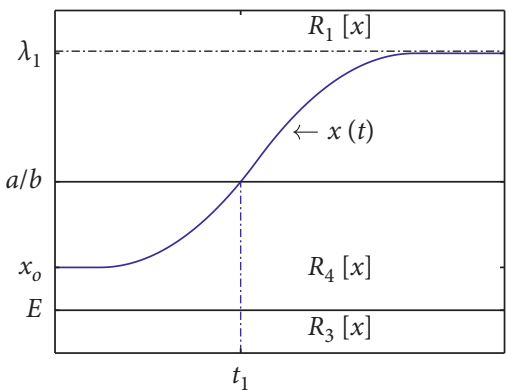

(c)

FIgURE 16: The behavior of the global trajectory $x(t)$ under the $\mathscr{H}[2,2,2]$ arrangement. (a) Population size begins in the region $\mathfrak{R}_{4}[x]$ following $x_{4}(t)$ with $\lambda_{4}>E$; if $E<x_{0}<\lambda_{4}<a / b$, population size increases as it approaches $\lambda_{4}$. (b) On the other hand, if $E<\lambda_{4}<x_{0}<a / b$, population size decreases approaching $\lambda_{4}$ asymptotically. (c) Whenever $\lambda_{4}>a / b, \lambda_{4}$ equilibrium lies within the region $\mathfrak{R}_{1}[x]$, and then, since $E<x_{o}<a / b$, population size increases according to rule $x_{4}(t)$ approaching $\lambda_{4}$ reaching the $a / b$ boundary at time $t_{1}$ switching to rule $x_{1}(t)$; then, since $\lambda_{1}>a / b$, population size increases asymptotically towards $\lambda_{1}$ staying within region $\mathfrak{R}_{1}[x]$. 


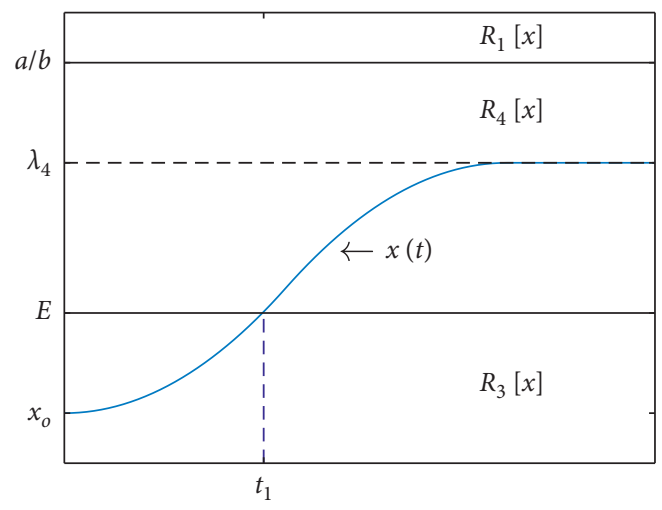

(a)

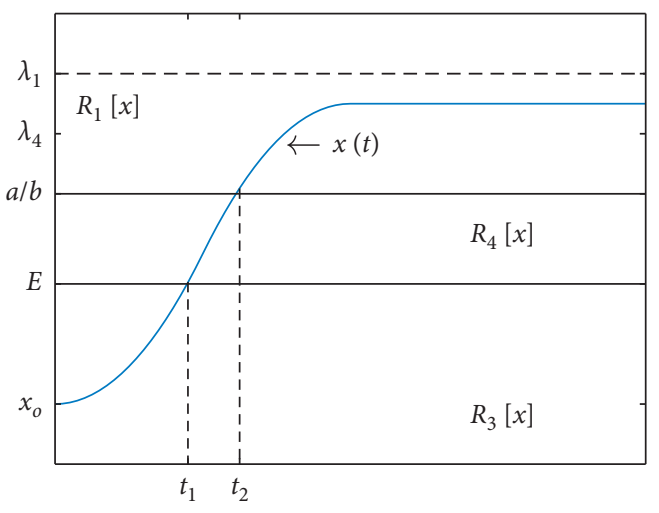

(b)

Figure 17: The form of the global trajectory $x(t)$ for the $\mathscr{H}[2,2,3]$ composite. (a) The initial condition $x_{o}<E$ sets population size $x(t)$ within the region $\mathfrak{R}_{3}[x]$ ruled by $x_{3}(t)$; continuity implies $x_{3}\left(t_{1}\right)=E$; then, for $t>t_{1}, x(t)$ increases according to $x_{4}(t)$ approaching $\lambda_{4}$ whenever this places in $\Re_{4}[x]$. (b) Whenever $\lambda_{4}$ places within region $\Re_{1}[x]$, by continuity, $x_{3}(t)$ reaches the $a / b$ boundary at a time $t_{2}$ switching to rule $x_{1}(t)$ satisfying $f_{1}(a / b)>0$ implying $\lambda_{1}>a / b$; therefore, $x(t)$ approaches $\lambda_{1}$ asymptotically.

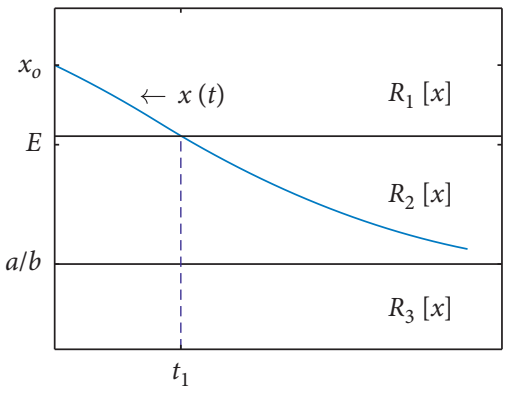

(a)

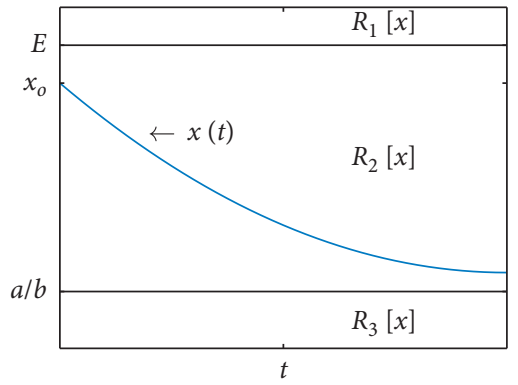

(b)

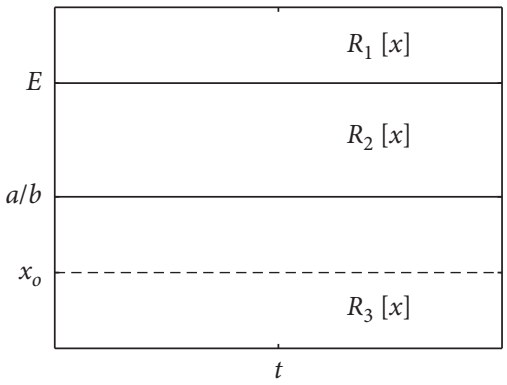

(c)

Figure 18: The construction of the global trajectory $x(t)$ for the $\mathscr{H}[1,3, \mathbf{k}]$ composite $(k=1,2,3)$. (a) For $\mathscr{H}[\mathbf{1}, \mathbf{3}, \mathbf{1}]$, population size starts at $x_{o}>E$ in region $\Re_{1}[x]$ and is controlled by $x_{1}(t)$ that bears the asymptotic limit $\lambda_{1}=\sqrt{a E / b}$ satisfying $a / b<\lambda_{1}<E<x_{0}$, and population size decreases reaching the $E$ threshold at a certain time $t_{1}$ switching to growing rule $x_{2}(t)$ and will asymptotically approach the equilibrium solution $\lambda_{2}=a / b$. (b) For the arrangement $\mathscr{H}[1,3,2]$, the initial condition placement $a / b<x_{0}<E$ sets population size behaving according to the $x_{2}(t)$ law in $\mathfrak{R}_{2}[x]$, and population size approaches the equilibrium solution $\lambda_{2}=a / b$. (c) Correspondingly, the $\mathscr{H}[1,3,3]$ composite sets $x_{o}<E$, and population size obeys the $x_{3}(t)$ rule in region $\mathfrak{R}_{3}[x]$, but since $\beta=\mu a$, it remains stationary at the $x_{o}$ value.

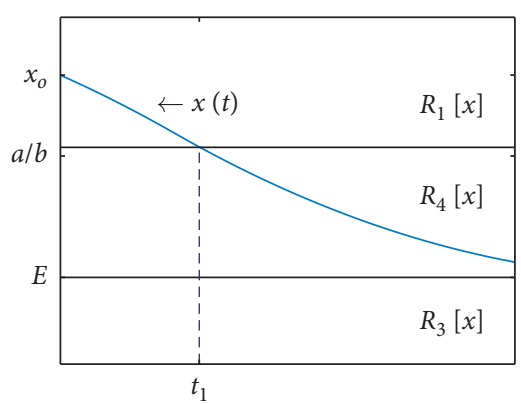

(a)

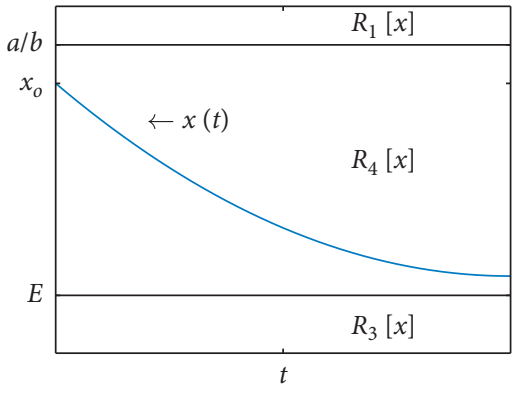

(b)

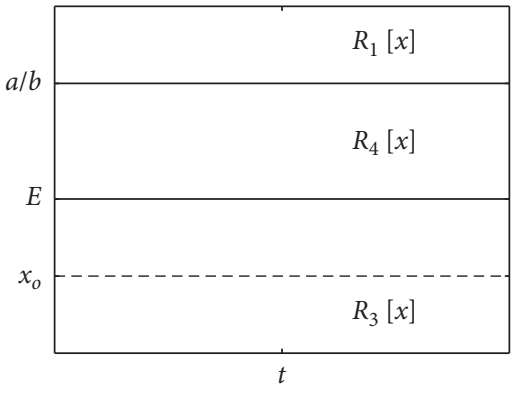

(c)

Figure 19: The assemblage of the global trajectory $x(t)$ for the $\mathscr{H}[2,3, \mathbf{k}]$ composite $(k=1,2,3)$. (a) For the $\mathscr{H}[\mathbf{2}, \mathbf{3}, \mathbf{1}]$ arrangement, population size $x(t)$ begins at $x_{o}>a / b$ within region $\mathfrak{R}_{1}[x]$ then $\lambda_{1}<a / b$ places in region $\Re_{4}[x], x(t)$ decreases, and intersects $a / b$ at a time $t_{1}$. Then switches to rule $x_{4}(t)$ which decreases and approaches the equilibrium solution $\lambda_{4}=E$. (b) For the case $\mathscr{H}[2,3,2]$, we have $E<x_{0}<a / b$, population size starts within the region $\mathfrak{R}_{4}[x]$ and sets by $x_{4}(t)$ approaching the $\lambda_{4}=E$ equilibrium. (c) For $\mathscr{H}[2,3,3]$, the condition $x_{0}<E$ and $\beta=\mu a$ set population size beginning within the region $\mathfrak{R}_{3}[x]$ and following $x_{3}(t)$ that remains stationary at $x_{0}$. 


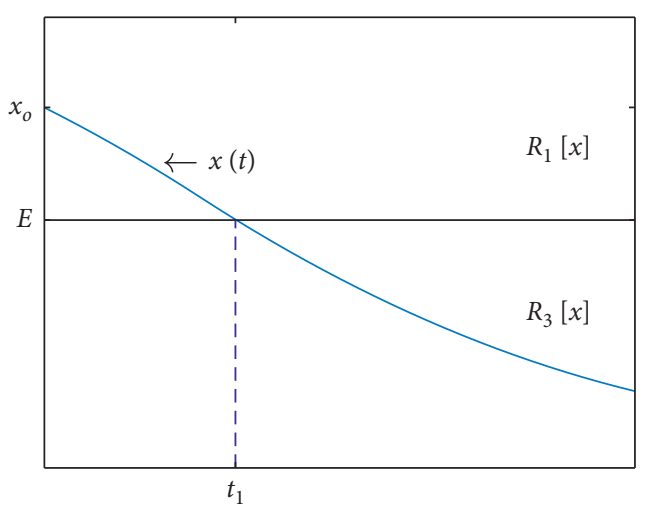

(a)

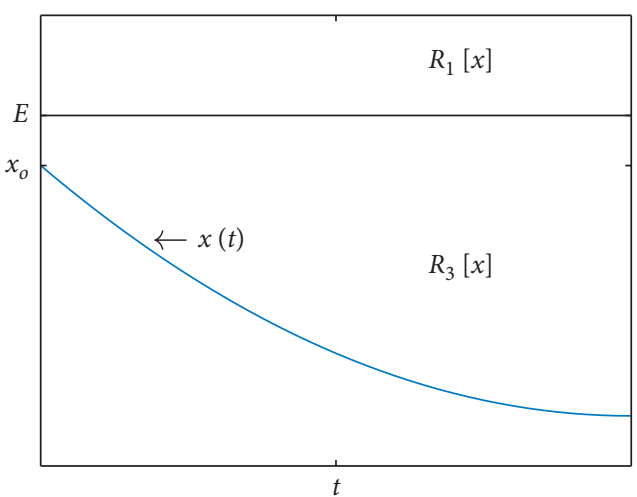

(b)

Figure 20: The form of the global trajectory $x(t)$ for the $\mathscr{H}[3,1, \mathbf{k}]$ composite $(k=1,2,3)$. (a) The case $\mathscr{H}[3,1,1]$ is associated to $x_{o}>E$; then, at the beginning, dynamics are set by the path $x_{1}(t)$ prevalent in region $\mathfrak{R}_{1}[x]$ that asymptotically approaches the value $\lambda_{1}<E$; then, population size decreases and hits the $E$ boundary at the time $t_{1}$ switching to $x_{3}(t)$ holding in region $\Re_{3}[x]$ which drives the population to extinction. (b) For the case $\mathscr{H}[3,1,2]$, the initial condition placement $x(0)<E$ keeps the population size within the region $\mathfrak{R}_{3}[x]$ as it progresses to extinction according to the $x_{3}(t)$ trajectory.

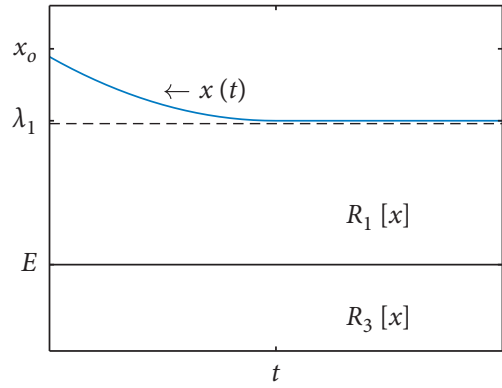

(a)

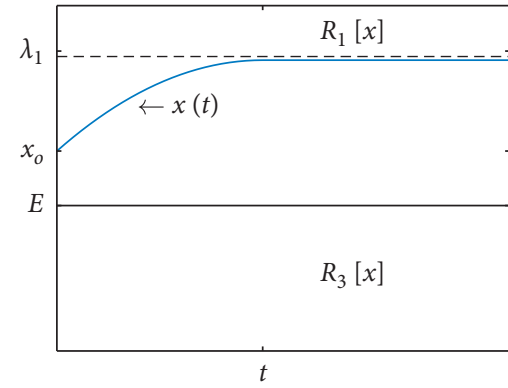

(b)

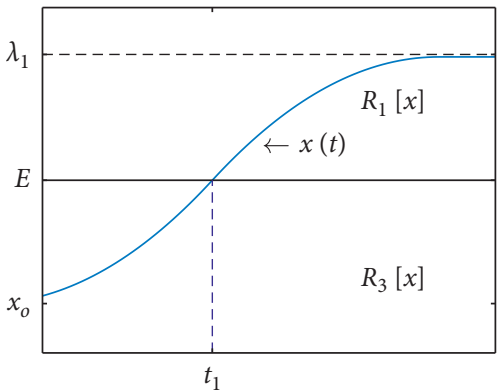

(c)

Figure 21: The global trajectory $x(t)$ for the $\mathscr{H}[3,2, \mathbf{k}]$ composite $(k=1,2,3)$. (a) For $\mathscr{H}[\mathbf{3}, \mathbf{2}, \mathbf{1}]$, we have $x_{o}>E$, so $x(t)$ begins in region $\mathfrak{R}_{3}[x]$, according to the $x_{1}(t)$ rule, so if $E<\lambda_{1}<x_{0}$, it asymptotically decreases approaching $\lambda_{1}>E$. (b) Alternatively, the $E<x_{0}<\lambda_{1}$ ordering sets population size increasing to $\lambda_{1}$. (c) For $\mathscr{H}[3,2,2]$, conditions $x(0)<E$ and $\beta>\mu a$ place $x(t)$ inside region $\Re_{3}[x]$ increasing according to $x_{3}(t)$ until it hits the $E$ boundary at a timet $t_{1}$, and then it switches to the $x_{1}(t)$ rule progressing towards the equilibrium solution $\lambda_{1}$ in $\Re_{1}[x]$.

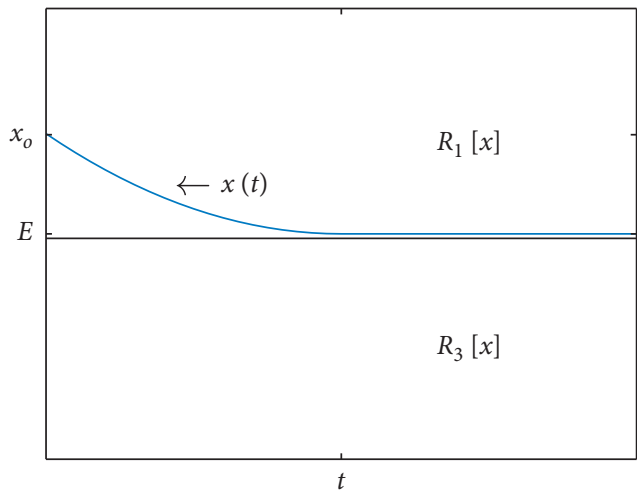

(a)

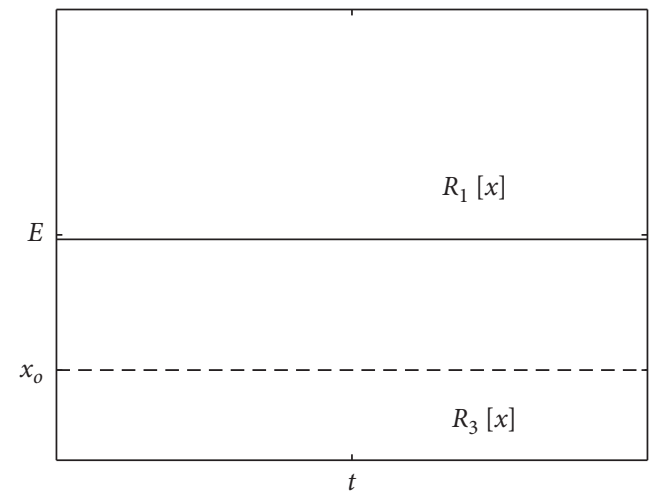

(b)

Figure 22: The comportment of the global trajectory $x(t)$ for the $\mathscr{H}[3,3, \mathbf{k}]$ composite $k=1,2,3)$. (a) For $\mathscr{H}[3,3, \mathbf{1}]$ condition, $x_{0}>E$ sets population size $x(t)$ to begin within the region $\mathfrak{R}_{1}[x]$ following $x_{1}(t)$. Since $\beta=u a$ and $E=a / b$ imply $\lambda_{1}=E$, then $x(t)$ decreases to $\lambda_{1}(b)$ For $\mathscr{H}[3,3,2]$, we have $x_{o}<E$, and population size starts within the region $\mathfrak{R}_{3}[x]$ and follows to growth law $x_{3}(t)$ but since $\beta=u a$, it remains stationary at $x_{0}$. 


$$
z_{n+1}= \begin{cases}\frac{s(1-r) E}{r}+z_{n}\left(1-r z_{n}\right), & \mathfrak{R}_{1}\left[z_{n}\right]=\left[\left(z_{n}>\frac{s E}{r}\right) \wedge\left(z_{n}>\frac{s a}{r b}\right)\right], \\ r z_{n}\left(1-z_{n}\right), & \mathfrak{R}_{2}\left[z_{n}\right]=\left[\left(z_{n}<\frac{s E}{r}\right) \wedge\left(z_{n}>\frac{s a}{r b}\right)\right], \\ \left(r-\frac{s a}{b}\right) z_{n}, & \mathfrak{R}_{3}\left[z_{n}\right]=\left[\left(z_{n}<\frac{s E}{r}\right) \wedge\left(z_{n}<\frac{s a}{r b}\right)\right], \\ \frac{s(r-1) E}{r}+\left(1-\frac{s a}{b}\right) z_{n}, & \mathfrak{R}_{4}\left[z_{n}\right]=\left[\left(z_{n}>\frac{s E}{r}\right) \wedge\left(z_{n}<\frac{s a}{r b}\right)\right] .\end{cases}
$$

For the sake of conciseness, we avert a detailed study of the performance of the discrete-time form of the PLF-PPM to an upcoming second part of this work. In the meantime, the parametrization of equation (25) given by equation (29) bears insight since it suggests that given the condition on the parameter $r$ that renders upset of chaos in the logistic map submodel $\theta_{2}\left(z_{n}\right)$, once the resulting orbit crosses the upper boundary of the region $\mathfrak{R}_{2}\left[z_{n}\right]$, dynamics will be set by the $\theta_{1}\left(z_{n}\right)$ submodel so the system will escape the chaotic regime and approach an equilibrium point in the region $\mathfrak{R}_{1}\left[z_{n}\right]$ or the $\theta_{1}\left(z_{n}\right)$ orbit could get back again to the chaotic regime determined by the logistic map $\theta_{2}\left(z_{n}\right)$. Alternatively, the $\theta_{2}\left(z_{n}\right)$ orbit could reach the lower boundary of the region $\Re_{2}\left[z_{n}\right]$ then switching to the $\theta_{3}\left(z_{n}\right)$ dynamics ruling in $\mathfrak{R}_{3}\left[z_{n}\right]$. Then depending in the paramter odering $z_{n}$ could decrease steadily and eventually vanish, or else wander aound in region $R_{3}\left[z_{n}\right]$ before getting back to the chaotic regime.

\section{Discussion}

Regulation by extreme value is observed in a plentiful of biological processes covering varied scales. It manifests on cellular structures such as mitochondria, where the maxima or minima of a periodical chemical reaction rule the formation of observed patterns [16]. Also, in the ecological settings, variables such as physical stress due to high or low temperatures, salinity, soil water content, wind velocities, and long or short exposures to air express better through extreme values than standard measures of central tendency $[17,18]$. The notion of biological control by extreme values dates back to Justus Von Liebig, who established the law of the minimum. It states that an organism's growth rate is regulated by the nutrient present in the minimum [5]. Generally, any factor that slows down potential growth in an ecosystem is described as a limiting factor. Acknowledgment of lower-upper tolerance limits for a process drove generalization of the law of the minimum into the law of the tolerance of Shelford [6].

Furthermore, the interaction of limiting factors can indirectly influence the effect of other factors not in themselves limiting. This paradigm, known as the principle of limiting factors, has proven to be very useful in studying whole or parts of ecosystems [13, 19]. The joint LiebigShelford paradigm adopted here bears that population

growth control occurs by balancing birth and mortality processes, the first determined by factor inducing the minimum offspring and the second by forcing the maximum number of deaths among individuals. This notion sustains the formal piecewise setup expressed by equation (22) and refers to PLF-PPM. Such a paradigm could allow a piecewise account of population dynamics composing growth phases delimited by density dependence cooperation and competition [20-23], thresholds of starvation, and critical density or extinction [20, 24]. The present PLF-PPM conceives growth phases controlled by limiting factors acting over domains bounded by population size thresholds. The first version of a PLF-PPM addressed a predator-prey model where Liebig's law governs the natality process of the prey population [1]. Echavarría and Gomez [25] and Echavarría et al. [26] extended these ideas to formulate models in which the mortality rate is set by the maximum value of factors that depend on population size. Montiel-Arzate et al. [3] adopted the referred Liebig-Shelford principle of limiting factors to formulate a functionally diverse population growth model. The approach in [3] relied on a parametrization of the birth process that rendered qualitative exploration burdensome, explaining why it is missing. Besides, Montiel-Arzate et al. [3] did not include a specific parametrization of mortality due to Allee effects which we incorporate here represented by the term $a x(t)$ as described by equation (21). Present settings allowed exhaustive qualitative exploration of the PLF-PPM global trajectory $x(t)$ presented in the Appendix. Even considering only population size and an external resource as limiting factors, the present formulation ensures a set of 20 basic orderings, each one associated to a different distribution of phases, which conform to an outstanding array of varying operation modes for the PLF-PPM. Such a flexible structure endures a sound interpretative strength displayed in the fitting results of the addressed study cases. Particularly, present settings allow visualization of abatement of resources controlling the birth process.

Nevertheless, a direct fitting procedure that relies on estimates' initial values brought high sensibility associated with local minimum problems at the non-linear acquisition of final values. These inconveniences suggest revision aimed to adapt a maximization of reproducibility strength criterion and simulation techniques that could lessen the parameter estimation burden experienced. Another point concerns adaptation of a symbolic manipulation code aimed at 
automatically exploring phase arrangement and their implications for stability. Yet another essential issue left untouched here concerns exploring the outlined discrete-time version of the PLF-PPM of equation (25) and its alternate form (29). In the meantime, the parametrization of equation (29) already suggests that even conditions on the parameter $r$ rendering upset of chaos in the logistic map submodel $\theta_{2}\left(z_{n}\right)$ once the resulting orbit crosses the upper boundary of the region $\Re_{2}\left[z_{n}\right]$ dynamics will be set by the $\theta_{1}\left(z_{n}\right)$ submodel, so the system will escape chaos before the orbit gets back again to the chaotic regime determined by the logistic map $\theta_{2}\left(z_{n}\right)$. Given the present results, no doubt that the suggested adaptation of codes to enhance both qualitative exploration and parameter fitting procedures will strengthen the applicability of offered PLF-PPM. Meanwhile, its present form already provides an outstanding research tool through which critical ecological parameters can be identified and be meaningfully interpreted.

\section{Conclusions}

This paper elucidates a logical deductive approach to establishing a piecewise structured model to interpret the growth of a single species population. The offered PLFPPM derives as a logical consequence of what we coined as a principle of limiting factors for population growth, a paradigm adapted by merging Liebig's law of the minimum and the tolerance law of Shelford. The formal approach explains by the extreme value characterization of the birth $N(x(t))$ and mortality $M(x(t))$ processes in equation (15). Conceived forms allow the selection of the factors that play the decisive role in controlling population growth at a given time. The flexibility from a piecewise structure imbues the PLF-PPM with a noticeable reproducibility strength. Considered study cases briefly elaborate on this. The PLFPPM bears as well an outstanding interpretative advantage. Such a feature infuses our construct with the capability of identifying different growth phases. These associate with regions of the dominance of crucial factors determining population dynamics such as a starvation regime, population size viability, or regimes of high population density effects. We only contemplated one external factor, $E$, and population size itself to describe $N(x(t))$ and $M(x(t))$. Concomitantly, the number of possible phase configurations was over ten times the number of parameters involved, which is the reason why ensuing parametrization proved to be seemingly adequate given addressed datasets. Nevertheless, as the number of explaining factors increases, more parameters are required. Then, the tied analytical exploration becomes complicated. Then particularly, acquiring suitable complexity could lead to local minimum difficulties connected to non-linear estimation. In this order of ideas, a quest for efficient parameter estimation methods that enhance the practical advantages derived from the PLF-PPM seems necessary. Such an endeavor concerns the research aims to pursue in a further paper. Another relevant research subject is pending, which is exhaustively exploring the discrete-time PLF-PPM construct outlined by equation (25).

\section{Appendix}

To study the diverse configurations of the phase portrait associated with the model of equation (22), we depart from the fundamental order relationship for the external energy $E$ and the ratio of mortality from population density proportions, $a / b$, that yields a first model type classification $\left(M_{i}, i=1,2\right)$. We have to consider model type $M_{1}$ associated to the inequality $E>a / b$, where the range of $x(t)$ composes the regions: $\mathfrak{R}_{1}[x]=\{a / b<E<x\}, \quad \mathfrak{R}_{2}[x]=\{a / b<x<E\}, \quad$ and $\mathfrak{R}_{3}[x]=\{x<a / b<E\}$. For model type $M_{2}$, where the relationship $E<a / b$ holds, the range of $x(t)$ will be divided into the regions $\mathfrak{R}_{1}[x], \mathfrak{R}_{3}[x]$, and $\mathfrak{R}_{4}[x]=\{a / b<x<E\}$. For model type $M_{3}$ linking to the ordering $E=a / b$, the range of $x(t)$ will be divided into the regions $\Re_{1}[x]$ and $\Re_{3}[x]$. Besides, it is also required to classify possible phase portrait arrangements according to the Birth to Mortality $a$-Scaled Ordering $\left(\mathrm{BMS}_{i}, i=1,2,3\right)$, namely, $\mathrm{BMS}_{1}$ : when $(\beta<\mu a), \mathrm{BMS}_{2}$ : if $(\beta>\mu a)$, and $\mathrm{BMS}_{3}$ : whenever $(\beta=\mu a)$. Finally, the placement of the initial condition placement determines a third classification pointer $\left(\mathrm{IC}_{\mathrm{ik}(i)}\right.$ with $i=1,2$ and $\left.k(i)=1,2, \ldots, 5\right)$, namely, $\mathrm{IC}_{11}$ for $\left(E<x_{0}\right), \mathrm{IC}_{12}$ : whenever $\left(a / b<x_{o}<E\right), \mathrm{IC}_{13}$ : whenever $\left(x_{o}<a / b\right), \mathrm{IC}_{14}$ : if $\left(x_{o}=E\right)$, and $\mathrm{IC}_{15}$ : whenever $\left(x_{o}=a / b\right)$. Correspondingly, $\mathrm{IC}_{21}$ : for $\left(x_{o}<E\right), \quad \mathrm{IC}_{22}$ : whenever $\left(E<x_{0}<a / b\right), \quad \mathrm{IC}_{23}$ : when $\left(x_{o}>a / b\right), \mathrm{IC}_{24}$ : if $\left(x_{o}=E\right)$, and $\mathrm{IC}_{25}$ : whenever $\left(x_{o}=a / b\right)$. The possible phase portrait arrangements typify according to a three-dimensional conjunction operator $\mathfrak{R}[i, j, k(i)]=\left(M_{\mathrm{i}} \wedge \mathrm{BMS}_{j} \wedge \mathrm{IC}_{\mathrm{ik}(i)}\right)$ for $i$ and $k(i)$ as specified. Tables 1 and 2 summarizes phase arrangements and corresponding parameter orderings.

\section{(A). Analysis of Trajectories for Orderings of the $\mathscr{H}[1,1, \mathbf{k}]$ Type}

We begin by analyzing case $\mathscr{H}[1,1,1]$ associated to conjunction $\left(M_{1} \wedge \mathrm{BMS}_{1} \wedge \mathrm{IC}_{11}\right)$ : model type $\left.M_{1}:(E>a / b)\right)$, Birth to Mortality Scaled Order $\left(\mathrm{BMS}_{1}\right):(\beta<\mu a)$, and initial condition ordering $\left(I C_{11}\right):\left(x_{o}>E\right)$. Given this parametric arrangement, the range $X_{R}=\cup_{i=1,2,3} \mathfrak{R}_{i}[x]$ is associated. Then, since $x_{o}>E$, at the beginning of the growth process, population size departs from a value $x(0)>E$ and its dynamics are set by the path $x_{1}(t)$ prevailing in region $\mathfrak{R}_{1}[x]$.

$$
x_{1}(t)=\frac{\lambda_{1}\left(C-e^{-2 t^{\sqrt{\beta \mu b E}}}\right)}{C+e^{-2 t^{\sqrt{\beta \mu b E}}}}, \quad \lim _{t \rightarrow \infty} x_{1}(t)=\lambda_{1},
$$

where $\lambda_{1}=\sqrt{\beta E / \mu b}$ and $C$ can be determined from the condition $x(0)=x_{o}$.

Besides, in $\Re_{1}[x]$, the growth law, $\dot{x}(t)=\beta E-\mu b x^{2}(t)$, applies; therefore, $\dot{x}(t)<0$ whenever $x(t)>\sqrt{\beta E / \mu b}$ and the trajectory $x(t)$ will monotonically decrease towards $\sqrt{\beta E / \mu b}$. Since we also have $\beta<\mu a$, then $\beta / \mu b<a / b<E$ which implies $\beta E / \mu b<E^{2}$, and thus the equilibrium placement $\sqrt{\beta E / \mu b}<E<x_{0}$ holds. Then, departing from $x_{0}$, the trajectory asymptotically decreases towards $\sqrt{\beta E / \mu b}<E$. But, by continuity, while $x_{1}(t)$ approaches $\sqrt{\beta E / \mu b}<E$, it will first hit the $E$ value at a particular time $t_{1}$, that is, $x_{1}\left(t_{1}\right)=E$ 
(see Figure 9(a))). Then, it keeps forward entering into the region $\mathfrak{R}_{2}[x]$ with dynamics switching to $x_{2}(t)$, namely,

$$
x_{2}(t)=\frac{\beta}{C e^{-\beta t}+\mu b} \text {, with, } \quad \lim _{t \longrightarrow \infty} x(t)=\frac{\beta}{\mu b},
$$

where $C$ can be determined from the condition $x\left(t_{1}\right)=E$.

Since $\dot{x}(t)$ is continuous, it turns out that $x(t)$ will keep decreasing. But, since $\beta<\mu a$, we have $\beta / \mu b<a / b<E$; besides, in region $\mathfrak{R}_{2}[x]$, we have $\dot{x}(t)=(\beta-\mu b x) x$; then, $\dot{x}(t)<0$ for $x>\beta / \mu b$. Therefore, population size will decrease asymptotically approaching the value $\beta / \mu b$. But under the inequality $\beta / \mu b<a / b$ and given the continuity of $x(\mathrm{t})$, there is a real number $t_{2}$ such that $x\left(t_{2}\right)=a / b$ (see Figure 9(b)). Next, $x(t)$ switches to the growth form $x_{3}(t)$ holding in region $\mathfrak{R}_{3}[x]$, namely,

$$
x_{3}(t)=x_{o} e^{(\beta-\mu a) t}, \text { with, } \quad \lim _{t \longrightarrow \infty} x(t)=0,
$$

and since $\beta<\mu a$, the population size decreases until it eventually becomes extinct. So, for the phase portrait arrangement $\mathscr{H}[\mathbf{1}, \mathbf{1}, \mathbf{1}]$, population size displays the global path shown in Figure 9(c).

We now undertake case $\mathscr{H}[\mathbf{1}, \mathbf{1}, \mathbf{2}]:\left(M_{1} \wedge \mathrm{BSM}_{1} \wedge \mathrm{IC}_{12}\right)$ : model type $M_{1}:(E>a / b)$, Birth to Mortality Scaled Order 1 $\left(\mathrm{BMS}_{1}\right):(\beta<\mu a)$, and initial condition ordering $\left(\mathrm{IC}_{12}\right):\left(a / b<x_{0}<E\right)$. Based on the previous analysis, we obtain that whenever $a / b<x_{0}<E$, population size $x(t)$ places initially within the region $\mathfrak{R}_{2}[x]$ according to the $x_{2}(t)$ law approaching the asymptotic limit $\beta / \mu b$. Since for the case $\mathscr{H}[1,1,2]$, the inequality $\beta / \mu b<a / b$ holds, population size decreases, so it eventually reaches the $a / b$ threshold at a time $t_{1}$ (see Figure 10(a)). After that, it enters the region $\mathfrak{R}_{3}[x]$ and again since $\beta<\mu a$, it will keep decreasing now according to the $x_{3}(t)$ law, thereby becoming extinct (see Figure 10(a)).

We now explain the performance of $x(t)$ in the case $\mathscr{H}[1,1,3]:\left(M_{1} \wedge \mathrm{BMS}_{1} \wedge \mathrm{IC}_{13}\right):$ model type $M_{1}:(E>a / b)$ and Birth to Mortality Scaled Order $1\left(\mathrm{BMS}_{1}\right):(\beta<\mu a)$ and initial condition ordering $\left(\mathrm{IC}_{13}\right):\left(x_{0}<a / b<E\right)$. For this arrangement, population size initially is placed within the region $\mathfrak{R}_{3}[x]$ and then evolves according to growth law $x_{3}(t)$, but since we have $\beta<\mu a$, it keeps decreasing until it eventually vanishes (see Figure 10(b)).

\section{(B). Analysis of Trajectories for Orderings of the $\mathscr{H}[2,1, \mathbf{k}]$ Type}

Let us now analyze the global trajectory $x(t)$ for an $\mathscr{H}[2,1,1]$ arrangement. We have that the initial condition placement $x_{o}>a / b>E$ applies. At the beginning of the growth process, population size $x(t)$ will take on a $x_{1}(t)$ form as given by equation (A.1) which decreases for $t \geq 0$ and asymptotically approaches a value $\sqrt{\beta E / \mu b}$. Now, for $\mathscr{H}[2,1,1]$, the statement $\beta<\mu a$ is true. Then, $\mu a / \beta>1$ implying $\mu a^{2} / \beta b>a / b$ and since parameters satisfy the condition defining model type $M_{2}$, we have $\mu a^{2} / \beta b>a / b>E$ and consequently $a^{2} / b>\beta E / \mu$, namely, $(a / b)^{2}>\beta E / \mu b$. Taking also into account that $\beta E / \mu b>0$, it follows that $a / b>\sqrt{\beta E / \mu b}$. Then, due to the continuity of $x(t)$ while approaching $\sqrt{\beta E / \mu b}$, there must be a real number $t_{1}$ such that $x\left(t_{1}\right)=a / b$ (see Figure 11(a)). Then, for $t \geq t_{1}$, dynamics will take place in the region $\mathfrak{R}_{4}[x]$, where population size behaves according to the $x_{4}(t)$ rule, namely,

$$
x_{4}(t)=x_{o} e^{-\mu a t}+\frac{\beta E}{\mu a}\left(1-e^{-\mu a t}\right) \text {, with } \lim _{t \rightarrow \infty} x(t)=\lambda_{4} \text {, }
$$

where $\lambda_{4}=\beta E / \mu a$. Since the condition $\beta<\mu a$ implies $\beta / \mu a<1$, then $\lambda_{4}<E$.

Inside $\mathfrak{R}_{4}[x]$, the dynamical system shapes $\dot{x}=\beta E-\mu a x$, and then $\dot{x}(t)<0$ for $x(t)>\beta E / \mu a$ and population size will decrease while asymptotically approaching the equilibrium solution $\beta E / \mu a$ placing below $E$, but on its way to reach $\beta E / \mu a$, by continuity, the $x(t)$ trajectory will necessarily cross the $E$ threshold at a specific time $t_{2}$. Once entering the region $\mathfrak{R}_{3}[x]$, population size will be controlled by $x_{3}(t)$ but since $\beta<\mu a$, the population eventually vanishes (see Figure 11(a)).

Considering the analysis above, the construction of the $x(t)$ path in the case $\mathscr{H}[2,1,2]$ follows through. The initial condition placement $E<x_{o}<a / b$ implies population entering the region $\mathfrak{R}_{3}[x]$ and again population becomes extinct (see Figure 11(b)). The $\mathscr{H}[\mathbf{2}, \mathbf{1}, \mathbf{3}]$ case encompasses the statement $\left(M_{2} \wedge \mathrm{BMS}_{1} \wedge \mathrm{IC}_{23}\right) \equiv 1$, so we have that the initial condition satisfies $x_{o}<E<a / b$. Therefore, population size starts within the region $\mathfrak{R}_{3}[x]$ and behaves according to rule $x_{3}(t)$, but again, since $\beta<\mu a$, the trend will be decreasing, and eventually, the population will become extinct (see Figure 11(c)). In summary, the $\mathscr{H}[\mathbf{i}, 1, \mathbf{k}]$ arrangements for $i=1,2$ and $k=1,2, \ldots, 5$ imply the population's extinction.

\section{(C). Analysis of Trajectories for Orderings of the $\mathscr{H}[1,2, \mathbf{k}]$ Type}

We address the case $\mathscr{H}[\mathbf{1}, \mathbf{2}, \mathbf{1}]:\left(M_{1} \wedge \mathrm{BMS}_{2} \wedge \mathrm{IC}_{21}\right)$ : model type $M_{1}:(E>a / b)$, Birth to Mortality Scaled Order $\left(\mathrm{BMS}_{2}\right):(\beta>\mu a)$, and initial condition ordering $\left(\mathrm{IC}_{11}\right):\left(x_{o}>E\right)$. Two additional orderings determine the phase arrangement. They are associated to the placement relative to $E$ of the equilibrium solution $\lambda_{2}=\beta / \mu b$ associated to the dynamical system prevailing in the region $\mathfrak{R}_{2}[x]$. We have to take into account the additional $(\beta / \mu b<E))$ and $\left.\left(\lambda_{2}>E\right)\right)$ orderings.

Assume $\left(\left(\lambda_{2}<E\right) \wedge \mathscr{H}[\mathbf{1}, \mathbf{2}, \mathbf{1}]\right) \equiv 1$. Since $x_{o}>E$, at the beginning of the process, population size will be set by $x_{1}(t)$ (see equation A.1), the law that prevails in $\mathfrak{R}_{1}[x]$, where $\lambda_{1}=$ $\sqrt{\beta E / \mu b}$ and $C$ can be determined from the condition $x(0)=x_{0}$. Besides, in $\Re_{1}[x]$, the dynamical system takes on the form, $\dot{x}(t)=\beta E-\mu b x^{2}$, that is associated to the equilibrium solution $\lambda_{1}=\sqrt{\beta E / \mu b}=\sqrt{\lambda_{2} E}$, and as we explained $\dot{x}(t)<0$ for $x(t)>\lambda_{1}$. Besides, the $\beta>\mu a$ ordering implies $\lambda_{2}>a / b$ or equivalently $\lambda_{2} E>a E / b>a^{2} / b^{2}$; then, $\lambda_{1}>a / b$. Since the additional ordering condition $\lambda_{2}<E$ applies, we would have $\lambda_{2} E<E^{2}$ which implies $E>\lambda_{1}>a / b$. Then, in its approach to $\lambda_{1}$, the $x_{1}(t)$ trajectory will hit the $E$ threshold, i.e., there exists a real number $t_{1}$ such that $x_{1}\left(t_{1}\right)=E$, and then for $t>t_{1}$, population size $x(t)$ turns to be controlled by 
$x_{2}(t)$ (see equation (A.2)). Therefore, the trajectory approaches asymptotically the equilibrium solution $\lambda_{2}$. But since we established $\lambda_{2}>a / b$, then $x_{2}(t)$ remains in region $\mathfrak{R}_{2}[x]$ (see Figure 12(a)).

If $\left(\left(\lambda_{2}>E\right) \wedge \mathscr{H}[\mathbf{1}, \mathbf{2}, \mathbf{1}]\right) \equiv 1$, then $\beta E / \mu b>E^{2}$ holds, implying $\lambda_{1}>E$; then, the condition $E<x_{0}<\lambda_{1}$ will set the $x(t)$ trajectory to increase according to the $x_{1}(t)$ rule, and it will approach the asymptotic limit $\lambda_{1}$ while staying within the region $\mathfrak{R}_{1}[x]$ (see Figure 12(b)). On the other hand, whenever we have $E<\lambda_{1}<x_{o}$, the population size $x(t)$ will decrease asymptotically towards $\lambda_{1}$ (see Figure 12(c)). Therefore, this establishes $\lambda_{1}$ as a stable equilibrium in the region $\Re_{1}[x]$.

We now consider case $\mathscr{H}[\mathbf{1}, \mathbf{2}, \mathbf{2}]:\left(M_{1} \wedge \mathrm{BMS}_{2} \wedge \mathrm{IC}_{12}\right)$ : model type $\left.M_{1}:(E>a / b)\right)$, Birth to Mortality Scaled order $\left(\mathrm{BMS}_{2}\right):(\beta>\mu a)$, and initial condition placing $\left.\left(\mathrm{IC}_{12}\right):\left(a / b<x_{o}<E\right)\right)$. Assume $\left(\left(\lambda_{2}>E\right) \wedge \mathscr{H}[1,2,2]\right) \equiv 1$. Under this conjunction, the $\beta>\mu a$ ordering implies $\lambda_{2}>a / b$, so we have $a / b<x_{o}<E<\lambda_{2}$. On the other hand, $\lambda_{2} E>a E / b$, and since $E>a / b$ implies $a E / b>a^{2} / b^{2}$, then we have $\lambda_{2} E>a^{2} / b^{2}$ or equivalently $\lambda_{1}>a / b$. Now since $\lambda_{2}>E$ applies, we would have $\lambda_{2} E>E^{2}$ which implies $a / b<E<\lambda_{1}$. Therefore, at the beginning of the growth process, population size places inside $\mathfrak{R}_{2}[x]$ and for $t>0$, it behaves according to the law $x_{2}(t)$ (see equation (A.2)).

For $x(t)<\lambda_{2}, \dot{x}(t)>0$, so population size will grow logistically in region $\Re_{2}[x]$ approaching the limit $\lambda_{2}$ that places in the region $\Re_{1}[x]$; therefore, for a specific value $t_{1}$, the statement $x\left(t_{1}\right)=E$ fulfills, and from that moment the population will grow logistically, according to growth law $x_{1}(t)$ approaching asymptotically to the $\lambda_{1}$ equilibrium (see Figure 13(a)).

Assume now that $\left(\left(\lambda_{2}>E\right) \wedge \mathscr{H}[\mathbf{1}, 2,2]\right) \equiv 1$. Since $\beta>\mu a$, the ordering $\lambda_{2}>a / b$ maintains; then, since $\lambda_{2}<E$, we could have $a / b<x_{0}<\lambda_{2}<E$ or $a / b<\lambda_{2}<x_{o}<E$. If the ordering $a / b<x_{0}<\lambda_{2}<E$ is true, the population size $x(t)$ will behave according to the $x_{2}(t)$ law, and then it will decrease from $x_{o}$ and approach the asymptotic limit $\lambda_{2}$. For $a / b<\lambda_{2}<x_{o}<E$, the population size $x(t)$ is still clinching to the $x_{2}(t)$ law and then will increase from $x_{o}$ and approach $\lambda_{2}$. Then, $\lambda_{2}$ shows a stable equilibrium (see Figure 13(b)).

We now consider case $\mathscr{H}[\mathbf{1}, \mathbf{2}, \mathbf{3}]:\left(M_{1} \wedge \mathrm{BMS}_{2} \wedge \mathrm{IC}_{13}\right)$ : model type $\left.M_{1}:(E>a / b)\right)$ and Birth to Mortality Scaled order $\left(\mathrm{BMS}_{2}\right):(\beta>\mu a)$ and initial condition placing $\left(\mathrm{IC}_{12}\right):\left(x_{0}<a / b<E\right)$. Assume also that $\left(\left(\lambda_{2}>E\right) \wedge \mathscr{H}[1,2,3]\right) \equiv 1$. Then, as we have explained the order relationship $x_{o}<a / b<E<\lambda_{2}$, the population size $x(t)$ places initially within the region $\mathfrak{R}_{3}[x]$ and grows according to the $x_{3}(t)$ trajectory (see equation (A.3)), which is the reason why the population will initiate an increasing exponential growth. When crossing by the value $x(t)=a / b$, the population will stop growing exponentially and will be governed by $x_{2}(t)$, the logistic law in $\Re_{2}[x]$, and will asymptotically approach the equilibrium solution $\lambda_{2}$. But since $E<\lambda_{2}$, eventually, population size will reach the level $E$ so dynamics will turn to be set by the $x_{1}(t)$ growing pattern, and it consequently will asymptotically approach the $\lambda_{1}$ threshold placed in the $\mathfrak{R}_{1}[x]$ region (see Figure 14(a)).
Similarly, the analysis of the conjunction $\left(\left(\lambda_{2}<E\right) \wedge \mathscr{H}[1,2,3]\right)$ is true. As the inequality $x_{0}<a / b<\lambda_{2}<E$ holds, once the population size reaches the $a / b$ boundary, it switches from the rule $x_{3}(t)$ to $x_{2}(t)$ and remains in region $\mathfrak{R}_{2}[x]$ approaching the asymptotic limit $\lambda_{2}$ (see Figure 14(b)).

\section{(D). Analysis of Trajectories for Orderings of the $\mathscr{H}[2,2, \mathbf{k}]$ Type}

We now consider the arrangement $\mathscr{H}[\mathbf{2}, \mathbf{2}, \mathbf{1}]$ : $\left(M_{2} \wedge \mathrm{BMS}_{2} \wedge \mathrm{IC}_{21}\right):$ model type $\left(M_{2}: E<a / b\right)$, Birth to Mortality Scaled order $\left(\mathrm{BMS}_{2}\right):(\beta>\mu a)$, and initial condition placing $\left(\mathrm{IC}_{21}\right):\left(x_{0}>a / b\right)$. The range of $x(t)$ will compose the regions $\mathfrak{R}_{3}[x], \mathfrak{R}_{4}[x]$, and $\mathfrak{R}_{1}[x]$. Then, $\beta>\mu a$ which leads to $\beta E / \mu b>b E / a$ but $E^{2}<a E / b i m p l y i n g ~ \lambda_{1}>E$. Then, since $x_{o}>a / b$, population size $x(t)$ begins within the region $\Re_{1}[x]$. If $E<a / b<\lambda_{1}<x_{0}$, population size decreases as it approaches $\lambda_{1}$ (see Figure 15(a)). On the other hand, if $E<a / b<x_{0}<\lambda_{1}$, population size increases approaching $\lambda_{1}$ asymptotically (see Figure 15(b)).

Now, since $\lambda_{1}>E$, this equilibrium solution could place such that $E<\lambda_{1}<a / b<x_{0}$. Again population size initiates dynamics within the region $\Re_{1}[x]$ and models according to the $x_{1}(t)$ rule. Therefore, population size $x(t)$ decreases towards $\lambda_{1}$, but since the whole trajectory $x(t)$ is continuous, there exists a real number $t_{1}$ such that $\left(t_{1}\right)=a / b$, so for $t>t_{1}$, the population now places within the region $\mathfrak{R}_{4}[x]$ and behaves according to rule $x_{4}(t)$ (see equation (A.4)), where $\lambda_{4}=\beta E / \mu a$. Moreover, $x_{4}(t)$ decreases whenever it attains values above $\lambda_{4}$ and increases if it places below $\lambda_{4}$. We have $\beta / \mu a>1$, and then $\lambda_{4}>E$. Therefore, $E<\lambda_{4}<a / b<x_{o}$ holds, and then $x(t)$ decreases as it approaches asymptotically $\lambda_{4}$ (see Figure 15(c)).

Assume that the $\mathscr{H}[\mathbf{2}, \mathbf{2}, \mathbf{2}]$ arrangement holds. Then, we have $E<a / b$, so the range of $x(t)$ composes regions $\mathfrak{R}_{3}[x], \mathfrak{R}_{4}[x]$, and $\mathfrak{R}_{1}[x]$. Since $E<x_{o}<a / b$, population size $x(t)$ initially places within the region $\mathfrak{R}_{4}[x]$ following the $x_{4}(t)$ law. But $\beta>\mu a$ implies $\beta E / \mu a>E$; therefore, $\lambda_{4}>E$. We have two possible orderings for the placement of $\lambda_{4}$ relative to $a / b$, namely, $O_{1}\left(\lambda_{4}, a / b\right):\left(E<\lambda_{4}<a / b\right)$ and $\mathrm{O}_{2}\left(\lambda_{4}, a / b\right):\left(E<a / b<\lambda_{4}\right)$. Additionally, conditioned on $O_{1}\left(\lambda_{4}, a / b\right) \equiv 1$, we have to take into account the location of $x_{0}$ comparative to $\lambda_{4}$. That is, $O_{11}\left(\lambda_{4}, x_{0}\right):\left(E<x_{o}\right.$ $\left.\left.<\lambda_{4}<a / b\right)\right)$ or $\left.O_{12}\left(\lambda_{4}, x_{o}\right):\left(E<\lambda_{4}<x_{o}<a / b\right)\right)$. Assume $\left(O_{11}\left(\lambda_{4}, x_{0}\right) \wedge \mathscr{H}[2,2,2]\right) \equiv 1$. Thus, $x(t)$ increases and approaches $\lambda_{4}$ staying within $\mathfrak{R}_{4}[x]$ (see Figure 16(a)). Undertake now $\left(O_{12}\left(\lambda_{4}, x_{o}\right) \wedge \mathscr{H}[\mathbf{2}, \mathbf{2}, \mathbf{2}]\right) \equiv 1$; accordingly, $x(t)$ starts above $\lambda_{4}$, so for $t>0$, population size will decrease while approaching asymptotically this value, again staying within $\mathfrak{R}_{4}[x]$ (see Figure 16(b)).

Whenever $\left(\left(\lambda_{4},<a / b\right) \wedge \mathscr{H}[\mathbf{2}, \mathbf{2}, \mathbf{2}]\right) \equiv 1$, the $\lambda_{4}$ equilibrium lies within the region $\Re_{1}[x]$. Then, since $E<x_{o}<a / b$, population size increases according to rule $x_{4}(t)$ approaching $\lambda_{4}$. Continuity of $x(t)$ implies the existence of a real number $t_{1}>0$ such that $x_{4}\left(t_{1}\right)=a / b$, so for $t>t_{1}$, population size models by rule $x_{1}(t)$. Now, we have $f_{4}(x)=\beta E-\mu a x(t)$; then, $f_{1}(a / b)=\beta E-\mu b(a / b)^{2}$. Additionally, by continuity of $f(x)$, it follows that 
$f_{1}(a / b)=f_{4}(a / b)$ and since we assumed $a / b<\lambda_{4}$, necessarily $f_{4}(a / b)>0$. Therefore, $f_{1}(a / b)>0$, that is, we also have $\beta E-\mu b(a / b)^{2}>0$ which in turn sets $\beta E>\mu b(a / b)^{2}$ or equivalently $\sqrt{\beta E / \mu b}>a / b$, that is, $\lambda_{1}>a / b$. Therefore, population size increases asymptotically towards $\lambda_{1}$ staying within region $\mathfrak{R}_{1}[x]$ for $t>t_{1}$ (see Figure 16(c)).

Whenever we have the $\mathscr{H}[\mathbf{2}, \mathbf{2}, \mathbf{3}]$ composite statement, the initial condition $x_{0}<E$ places population size $x(t)$ within the region $\Re_{3}[x]$ obeying the exponential growth law $x_{3}(t)$, and since $\beta>\mu a$, it will increase for $t>0$. This way, continuity of $x_{3}(t)$ implies the existence of a time $t_{1}$ such that $x_{3}\left(t_{1}\right)=E$. Then, for $t>t_{1}$, the behavior of $x(t)$ sets through $x_{4}(t)$. Again the statement $\beta>\mu a$ implies $\lambda_{4}>E$, so we could have $\left(\left(E<\lambda_{4}<a / b\right)\right.$ or $\left(E<a / b<\lambda_{4}\right)$ orderings. Assume $\left(\left(E<\lambda_{4}<a / b\right) \wedge \mathscr{H}[\mathbf{2}, \mathbf{2}, \mathbf{2}]\right) \equiv 1$. Then, $x(t)$ initiates in $\Re_{3}[x]$ and increases as it approaches $\lambda_{4}$ staying within $\mathfrak{R}_{4}[x]$. (see Figure 17(a)). Undertake now $\left(\left(E<a / b<\lambda_{4}\right) \wedge \mathscr{H}[\mathbf{2}, \mathbf{2}, \mathbf{2}]\right) \equiv 1$. Then, the $\lambda_{4}$ equilibrium places within the region $\Re_{1}[x]$ so by continuity, population size reaches the $a / b$ boundary switching to rule $x_{1}(t)$ and satisfying $f_{1}(a / b)>0$ which implies $\lambda_{1}>a / b$. Therefore, $x(t)$ approaches $\lambda_{1}$ asymptotically (see Figure 17(b)).

\section{(E). Analysis of Trajectories for Orderings of the $\mathscr{H}[1,3, \mathbf{k}]$ Type}

We begin by analyzing case $\mathscr{H}[\mathbf{1}, \mathbf{3}, \mathbf{1}]$ associated to conjunction $\left(M_{1} \wedge \mathrm{BMS}_{1} \wedge \mathrm{IC}_{11}\right)$ : model type $\mathrm{M}_{1}:(E>a / b)$ and Birth to Mortality Scaled Order $\left(\mathrm{BMS}_{3}\right):(\beta=\mu a)$ and initial condition ordering $\left(\mathrm{IC}_{11}\right):\left(x_{0}>E\right)$. Given this parametric arrangement, at the beginning of the growth process, population size departs from a value $x(0)>E$, and its dynamics are set by the path $x_{1}(t)$ (see equation (A.1)) prevailing in region $\Re_{1}[x]$, with $\lambda_{1}=\sqrt{\beta E / \mu b}$. Since $\beta=\mu a$, we have $\lambda_{1}=\sqrt{a E / b}$, but we also have $E>a / b$; then, $E^{2}>a E / b$, and therefore we obtain $\lambda_{1}<E$. On the other hand, if $E>a / b$, we also have $a E / b>(a / b)^{2}$ and consequently $\lambda_{1}>a / b$. Therefore, $a / b<\lambda_{1}<E<x_{0}$, and population size $x(t)$ decreases approaching $\lambda_{1}$. But since $\lambda_{1}$ lies in region $\mathfrak{R}_{2}[x]$, eventually population size will cross the $E$ threshold at a particular time $t_{1}$ and will switch to growing rule $x_{2}(t)$. This law is associated with the equilibrium solution $\lambda_{2}=a / b$, so population asymptotically approaches this value (see Figure 18(a)).

For the arrangement $\mathscr{H}[1,3,2]$, we have the initial condition placement $a / b<x_{o}<E$. Then, population size begins within the region $\Re_{2}[x]$ and behaves according to the $x_{2}(t)$ law. Therefore, population size approaches the equilibrium solution $\lambda_{2}=\beta / \mu b$, but since $\beta=\mu a$, we have $\lambda_{2}=a / b$. Then, population size approaches asymptotically the $a / b$ boundary (see Figure 18(b)). In turn, the $\mathscr{H}[1,3,3]$ composite encompasses the initial condition placement $x_{o}<E$; therefore, population size obeys the $x_{3}(t)$ rule, and since $\beta=\mu a$, it remains stationary at the $x_{o}$ value (see Figure 18(c)).

\section{(F). Analysis of Trajectories for Orderings of the $\mathscr{H}[2,3, \mathbf{k}]$ Type}

We now consider the arrangement $\mathscr{H}[\mathbf{2}, \mathbf{3}, \mathbf{1}]$ : $\left(M_{2} \wedge \mathrm{BMS}_{3} \wedge \mathrm{IC}_{21}\right):$ model type $\left.M_{2}:(E<a / b)\right)$, Birth to
Mortality Scaled order $\left(B M S_{3}\right):(\beta=\mu a)$, and initial condition placing $\left.\left(\mathrm{IC}_{21}\right): x_{0}>a / b\right)$. The range of $x(t)$ will compose the regions $\mathfrak{R}_{3}[x], \mathfrak{R}_{4}[x]$, and $\mathfrak{R}_{1}[x]$ Then, since $x_{o}>a / b$, population size $x(t)$ begins within the region $\Re_{1}[x]$ and hangs onto the $x_{1}(t)$ trajectory. Now, the $E<a / b i m p l i e s ~ a E / b<a^{2} / b^{2}$ and since $\beta=\mu a$, we have $\lambda_{1}=\sqrt{a E / b}$; therefore, $\lambda_{1}<a / b$. Consequently, $\lambda_{1}$ places in region $\Re_{4}[x]$, and the $x_{1}(t)$ trajectory decreases, so on its approach to $\lambda_{1}$, it will intersect the $a / b$ boundary at a time $t_{1}$. Subsequently, $x(t)$ switches to the rule $x_{4}(t)$ valid in $\Re_{4}[x]$. Now, the $\beta=\mu a$ setting implies $\lambda_{4}=E$, so since by continuity, we have $x_{4}\left(t_{1}\right)=a / b$, and population size decreases and approaches the equilibrium solution $\lambda_{4}$ matching the $E$ threshold (see Figure 19(a)). The case $\mathscr{H}[\mathbf{2}, \mathbf{3}, \mathbf{2}]$ is associated to the conjunction $\left(M_{2} \wedge \mathrm{BMS}_{3} \wedge \mathrm{IC}_{22}\right)$, so initial value $x_{o}$ fulfils $E<x_{0}<a / b$, and then population size starts within the region $\mathfrak{R}_{4}[x]$ and holds to growth law $x_{4}(t)$ approaching the $\lambda_{4}=\beta E / \mu a$ equilibrium which under the condition $\beta=\mu a$ satisfies $\lambda_{4}=E$. Therefore, $x(t)$ decreases asymptotically to $E$ (see Figure 19(b)). Finally, for $\mathscr{H}[2,3,3]$, the condition $x_{0}<E$ sets population size beginning within the region $\mathfrak{R}_{3}[x]$ following the $x_{3}(t)$ growth law. Again, the condition $\beta=\mu a$ sets $x_{3}(t)$ to remain stationary at $x_{0}$ (see Figure 19(c)).

\section{(G). Analysis of Trajectories for Orderings of the $\mathscr{H}[3,1, \mathbf{k}]$ Type}

We begin by analyzing case $\mathscr{H}[\mathbf{3}, \mathbf{1}, \mathbf{1}]$ associated to conjunction $\left(M_{3} \wedge \mathrm{BMS}_{1} \wedge \mathrm{IC}_{31}\right)$ : model type $M_{3}:(E=a / b)$ and Birth to Mortality Scaled Order $\left(\right.$ BMS $\left._{1}\right):(\beta<\mu a)$ and initial condition ordering $\mathrm{IC}_{31}:\left(x_{0}>E\right)$. Given this parametric ordering, population size confines only to regions $\Re_{1}[x]$ and $\mathfrak{R}_{3}[x]$. At the beginning of the growth process, population size departs from a value $x(0)>E$ and its dynamics are set by the path $x_{1}(t)$ prevalent in region $\Re_{1}[x]$, so it asymptotically approaches the value $\lambda_{1}=\sqrt{\beta E / \mu b}$. Again, the $\beta<\mu a$ ordering implies $\sqrt{\beta E / \mu b}<E$, and then population size decreases and eventually hits the $E$ boundary following the $x_{3}(t)$ rule holding in the region $\mathfrak{R}_{3}[x]$ which since $\beta<\mu a$ drives the population to extinction (see Figure 20(a)). The analysis of the case $\mathscr{H}[3,1,2]$ is straightforward since the initial condition placement $x(0)<E$ keeps the population size cling to region $\Re_{3}[x]$ where it progresses to extinction according to the $x_{3}(t)$ trajectory (see Figure 20(b)).

\section{(H). Analysis of Trajectories for Orderings of the $\mathscr{H}[3,2, \mathbf{k}]$ Type}

Whenever the $\mathscr{H}[3,2,1]$ ordering achieves, we have to consider the conjunction $\left(M_{3} \wedge \mathrm{BMS}_{2} \wedge \mathrm{IC}_{31}\right)$ : model type $M_{3}:(E=a / b)$, Birth to Mortality Scaled Order $\left(\mathrm{BMS}_{1}\right):(\beta>\mu a)$, and initial condition ordering $\left(\mathrm{IC}_{31}\right)$ : $\left(x_{0}>E\right)$. This way, population size $x(t)$ begins in region $\mathfrak{R}_{1}[x]$, and thus it obeys $x_{1}(t)$ rule, so it asymptotically approaches the value $\lambda_{1}=\sqrt{\beta E / \mu b}$. Correspondingly, the $\beta>\mu a$ ordering implies $\lambda_{1}>E$, and then population size decreases to $\lambda_{1}$ whenever $E<\lambda_{1}<x_{0}$ (see Figure 21(a)). On the other hand, whenever $E<x_{0}<\lambda_{1}$, population size increases to $\lambda_{1}$ (see Figure 21(b)). 
Similarly, for the case $\mathscr{H}[\mathbf{3}, \mathbf{2}, \mathbf{2}]$, the range of $x(t)$ composes the regions $\mathfrak{R}_{3}[x]$ and $\mathfrak{R}_{1}[x]$. The initial condition $x(0)<E$ places population size initially inside the region $\Re_{3}[x]$. The ordering $\beta>\mu a$ sets population size $x(t)$ to increase according to the $x_{3}(t)$ trajectory keeping inside the region $\Re_{3}[x]$ until it hits the $E$ boundary at a time $t_{1}$. Later, it switches to the $x_{3}(t)$ rule progressing towards the equilibrium solution $\lambda_{1}$ (see Figure 21(c)).

\section{(I). Analysis of Trajectories for Orderings of the $\mathscr{H}[3,3, \mathbf{k}]$ Type}

The $\mathscr{H}[3,3,1]$ ordering links to conjunction $\left(M_{3} \wedge \mathrm{BMS}_{2} \wedge \mathrm{IC}_{31}\right):$ model type $M_{3}:(E=a / b)$ and Birth to Mortality Scaled Order $\left(\mathrm{BMS}_{3}\right):(\beta=\mu a)$ and initial condition ordering $\left(\mathrm{IC}_{31}\right):\left(x_{o}>E\right)$ The range of $x(t)$ will compose the regions $\Re_{3}[x]$ and $\mathfrak{R}_{1}[x]$. Then, population size $x(t)$ begins within the region $\Re_{1}[x]$ and follows the $x_{1}(t)$ trajectory. Now, $\beta=\mu a$ and $E=a / b$ imply $\lambda_{1}=E$. Consequently, if $E=a / b=\lambda_{1}<x_{0}$, then $x(t)$ decreases as it approaches $\lambda_{1}$ (see Figure 22(a)).

The case $\mathscr{H}[3,3,2]$ is associated to the conjunction $M_{3} \wedge \mathrm{BMS}_{3} \wedge \mathrm{IC}_{32}$ so initial population size $x_{o}$ satisfies $x_{o}<E$. Then, population size starts within the region $\mathfrak{R}_{3}[x]$ and embraces to growth law $x_{3}(t)$ which under the condition $\beta=u a$ remains stationary at $x_{o}$. Therefore, $x(t)$ decreases asymptotically to $E$ (see Figure $22(\mathrm{~b})$ ). Similarly, the $\mathscr{H}[3,3,3]$ arrangement corresponds to the case $\mathscr{H}[3,3,2]$.

\section{Data Availability}

Data sources are referenced in the text.

\section{Conflicts of Interest}

The authors declare that there are no conflicts of interest regarding the publication of this paper.

\section{Acknowledgments}

This research was supported solely on institutional funding (CICESE).

\section{References}

[1] I. A. Polyetayev, "Modeli volterra, jishnir-zhertva y nekotorye ij obobschenyas ispolzovainien printsipa liebija," Zhurnal Obshchei Biologii, vol. 34, no. 1, p. 43, 1971.

[2] H. A. Echavarría and A. G. Gómez, "Modelos tipo volterra y el principio ecológico de los factores limitantes," Comunicaciones Internas, Facultad de Ciencias UNAM, vol. 6, pp. 1-25, 1975.

[3] E. Montiel-Arzate, H. Echavarría-Heras, and C. Leal-Ramírez, "A functionally diverse population growth model," Mathematical Biosciences, vol. 187, no. 1, pp. 21-51, 2004.

[4] W. C. Allee, Animal Aggregations, The University of Chicago Press, Chicago, IL, USA, 1931.

[5] L. Von and L. R. Pomeroy, "Principles of agricultural chemistry with special reference to the late researches made in
England," in Cycles of Essential Elements (Benchmark papers in Ecology), vol. Ip. 11, Dowwden, Hutchison \& Ross, Stroudsburg, PA, USA, 1974.

[6] V. E. Shelford, Animal Communities in Temperate North America, The University of Chicago Press, Chicago, IL, USA, 1913.

[7] J. T. Armstrong, "The population dynamics of the planarian, Dugesia Tigrina," Ecology, vol. 45, no. 2, pp. 361-365, 1964.

[8] J. Huisman, "Population dynamics of light-limited phytoplankton: microcosm experiments," Ecology, vol. 80, no. 1, pp. 202-210, 1999.

[9] J. Davidson, "On the growth of the sheep population in Tasmania," Transactions of the Royal Society of South Australia, vol. 62, pp. 342-346, 1938.

[10] R. Pearl, "The growth of populations," The Quarterly Review of Biology, vol. 2, no. 4, pp. 532-548, 1927.

[11] T. P. Hughes and J. E. Tanner, "Recruitment failure, life histories, and long-term decline of caribbean corals," Ecology, vol. 81 , no. 8 , pp. $2250-2263,2000$.

[12] L. I.-K. Lin, "A concordance correlation coefficient to evaluate reproducibility," Biometrics, vol. 45, no. 1, pp. 255-268, 1989.

[13] E. P. Odum, Fundamentals of Ecology, W.B. Saunders Co, Philadelphia, PA, USA, 3rd edition, 1971.

[14] C. J. Krebs, Ecology: The Experimental Analysis of Distribution and Abundance, Addison-Wesley, New York, NY, USA, 4th edition, 1994.

[15] J. H. Sang, "Population growth in Drosophila cultures," Biological Reviews, vol. 25, no. 2, pp. 188-219, 1950.

[16] A. E. R. Woodcock and M. Davis, Catastrophe Theory, E. P. Dutton, New York, USA, 1978.

[17] S. D. Gaines and M. W. Denny, "The largest, smallest, highest, lowest, longest, and shortest: extremes in ecology," Ecology, vol. 74, no. 6, pp. 1677-1692, 1993.

[18] M. W. Denny and S. O. Deines, "On the prediction of maximal intertidal wave forces," Limnology \& Oceanography, vol. 35, no. 1, pp. 1-15, 1990.

[19] E. P. Odum, Ecology, Holt Rinehart \& Winston, New York, NY, USA, 1963.

[20] H. T. Odum and W. C. Allee, "A note on the stable point of populations showing both intraspecific cooperation and disoperation," Ecology, vol. 35, no. 1, pp. 95-97, 1954.

[21] A. Nicholson, "An outline of the dynamics of animal populations," Australian Journal of Zoology, vol. 2, no. 1, pp. 9-65, 1954.

[22] W. M. Getz, "Population dynamics: a per capita resource approach," Journal of Theoretical Biology, vol. 108, no. 4, pp. 623-643, 1984.

[23] W. M. Getz, "A hypothesis regarding the abruptness of density dependence and the growth rate of populations," Ecology, vol. 77, no. 7, pp. 2014-2026, 1996.

[24] B. Dennis, "Allee effects: population growth, critical density, and the chance of extinction," Natural Resource Modeling, vol. 3, no. 4, pp. 481-538, 1989.

[25] H. A. Echavarría and A. G. Gómez, "El principio de los factores limitantes y el crecimiento de poblaciones," Comunicaciones Internas, Departamento de Matemáticas, UNAM, vol. 13, pp. 1-36, 1979.

[26] H. A. Echavarría, E. Solana, and H. Madrid, "A functionally diverse model for predator prey systems Lamberson," in Natural Resource Modeling and Management I, International Workshop for Natural Resource Modeling and Analysis, H. Echavarría and R. H. Lamberson, Eds., p. 47, Department of Ecology CICESE, Ensenada, Mexico, 1994. 